\title{
Ocean response to mesoscale atmospheric forcing
}

\author{
By ISIDORO ORLANSKI and L. J. POLINSKY, Geophysical Fluid Dynamics Laboratory/NOAA, \\ Princeton University, Princeton, New Jersey 08540, USA
}

(Manuscript received March 30, 1982; in final form January 4, 1983)

\begin{abstract}
Many processes have been proposed as possible forcing mechanisms for mesoscale oceanic variability. The present study shows that atmospheric forcing can be an important source of mesoscale variability in the ocean. We show that the response is linearly proportional to the product of the time scale of the storm and its intensity. We clarify the point that for storms with scales considerably smaller than the barotropic Rossby radius of deformation, the oceanic stratification and the horizontal extent of the storm are the only factors determining the penetration depth of the response, implying that it is not the Rossby radius of deformation but rather the scale of penetration depth $(h=(f / N) L)$ that characterizes the response.

In exploring the effect of differing eddy-viscosity parametization on oceanic response, we find no significant qualitative differences, although as one might expect we find quantitative differences in the results.

The role of the mixed layer is considered very important in the transfer of surface stresses down into the system. The mixed layer does not seem to be important in determining the characteristic lengths of the problem, however, at least for storms that give a penetration depth considerably larger than the mixed layer (for a mixed layer on the order of $20 \mathrm{~m}$, the storm should be larger than a few kilometers).

The non-linear advection terms seem to affect the adjustment process more by reducing the associated wave energy than by modifying the characteristics of the geostrophic response.

Finally, making the stratification more realistic has no significant impact on the resulting oceanic response.
\end{abstract}

\section{Introduction}

The routine coverage of the world ocean surface by satellites has shown a considerable number of highly variable small-scale surface disturbances, including eddies, fronts, and long internal gravity waves. Although the variability of the ocean has come as no surprise to oceanographers, its short time evolution was not previously inferred. The large variance found in the currents and temperature was formerly thought to be a consequence of processes generated internally by means of instabilities. More recently, however, the role of atmospheric forcing has become more apparent, not only on planetary scales but also on the mesoscale. The accepted definition of mesoscale comprises flow from less than $2000 \mathrm{~km}$ to almost the cloud scales or a few kilometers (Orlanski, 1975). The failure to recognize the importance of mesoscale atmospheric forcing in the production of long-lasting features in the ocean was primarily due to the time-scale disparity between mesoscale atmospheric phenomena and their oceanic counterparts. In fact, just how long it takes for the atmosphere to establish a geostrophically balanced flow in the ocean is the key question.

Atmospheric phenomena, by their highly turbulent nature, may decay very quickly because of the short time scale of turbulence, whereas an ocean current after being established can survive for a long time because of the lack of diffusive processes. We may ask then, what is the importance of mesoscale disturbances generated in the upper layers of the ocean? The effective mixing of the turbulent boundary layer in the ocean extends only a few tens of meters. Underneath this mixed layer the most recently revised turbulent diffusivity values have been shown to be very, very small, of 
the order of less than $10^{-4} \mathrm{~m}^{2} \mathrm{~s}^{-1}$, or a decay time scale of 1000 days for thermocline-scale depths. Therefore, surface mesoscale disturbances with penetrations deeper than the mixed layer may be the most efficient mechanism to produce effective transfers to the interior of the ocean. It has been suggested by many scientists (Nihoul, 1980) that surface oceanic fronts, a common feature in the ocean, may provide a means by which vertical transport can be achieved in the upper layers of the ocean. It then becomes relevant to ask, what are the processes by which mesoscale features in the ocean can be generated? As stated above, the idea of instabilities in ocean currents is appealing, but only perhaps in selected regions, such as over strong boundary currents. The process of frontogenesis by deformation fields, which is similar to the process which governs atmospheric fronts, has been suggested to explain those which occur in the ocean. However, the time scale required is of the order of a few weeks for the ocean, while atmospheric forcing takes only a few days to generate the same phenomena. It is therefore our intention to show in this paper that atmospheric forcing in scales of the order of $100 \mathrm{~km}$ can be very effective in explaining mesoscale oceanic variability. We shall also discuss, by using numerical and analytical solutions, the horizontal and vertical penetration scales of the oceanic response as a function of the external forcing and internal parameters, such as stratification, eddy viscosity, etc.

We will devote part of our effort to clarifying some aspects of the classical geostrophic adjustment problem. In particular, we shall address the question of what, if any, role the internal Rossby radius of deformation plays in selecting the scale of the response in a stratified ocean, and also the question of the depth of penetration of such a response. Since the geostrophic adjustment problem was first discussed by Rossby $(1937,1938)$, a great number of works have been devoted to that problem, basically to try to answer under which conditions a geostrophic flow will experience an increase in energy due to an impulsive imbalance. The crucial question has been to determine how much of the total energy remains in the geostrophic mode and how much is dissipated in wave energy. A different approach from the initial-value problem would be to consider the oceanic response to a surface forcing applied over a finite time. The latter, the boundary-value problem, has the advantage of introducing a time scale into the problem. Also, it can be treated under more realistically balanced conditions than the initial-value problems. A complete review of this subject, where both systems are discussed, can be found in Blumen (1972). Since the major results of previous works are well known, we will not review them here. As we have stated, however, we will present a discussion of the permanent features of the oceanic response to one of the most common atmospheric mesoscale phenomena, a cold front.

The governing equations with generalized at mospheric forcing in a stratified ocean with a mixed layer are derived in Section 2. The inverse Laplace Fourier transform of the solution in a constantly stratified ocean is discussed in Section 3.1, and the steady contribution from a transient stress is discussed in Section 3.2. The particular response for a homogeneous ocean and for the stratified case can be found in Sections 3.3 and 3.4, respectively. The discussion of the penetration depth as a function of the horizontal scale can be found in Section 3.5. In Section 4 the complete discussion of a two-dimensional numerical solution for the linear regime is presented. Note that the transient behaviour is discussed in Section 4.1; differences in the response to changes in the stratification are described in Section 4.2; the dependence of the response on the atmospheric forcing is covered in Section 4.3; and the dependence of the response on different eddy viscosities is presented in Section 4.4. Finally, the fully non-linear solutions for this particular case for constant and realistic stratifications are presented in Section 5.

\section{The linear problem}

The ocean response to idealized atmospheric forcing will be discussed with an analytic model which has the following constraints:

\subsection{Perturbation equations}

Consider a two-layer ocean in which the upper layer is homogeneous, with constant density $\rho_{0}$, and the lower one is continuously stratified, with $\rho=$ $\bar{\rho}(z)$. This structure corresponds roughly to an ocean with a mixed layer at the top in which momentum transfer occurs through wind stresses applied at the surface. The ocean is assumed to be 
infinite in the horizontal $(x, y)$ plane and rotating about a vertical axis. It is supposed that the water is initially at rest and that the subsequent motions are small. Then the response of the ocean to atmospheric pressure fluctuation and wind stresses at the surface will be described by:

$\frac{\partial u_{\mathrm{m}}}{\partial t}-f v_{\mathrm{m}}=-\frac{\boldsymbol{P}_{\mathrm{mx}}}{\rho_{0}}+\frac{\tau^{x}}{\rho_{0} D}$,

$\frac{\partial v_{\mathrm{m}}}{\partial t}+f u_{\mathrm{m}}=-\frac{\boldsymbol{P}_{\mathrm{m}_{\mathrm{y}}}}{\rho_{0}}+\frac{\tau^{y}}{\rho_{0} D}$,

$P_{\mathrm{m}}=g \rho_{0} \eta+P_{\mathrm{a}}$,

and

$$
w_{\mathrm{S}}-w_{\mathrm{B}}=-D \nabla_{H} \cdot \mathbf{V}_{\mathrm{m}} \text {, }
$$

in the mixed layer $(-D<z<0)$, where $u_{\mathrm{m}}$ and $v_{\mathrm{m}}$ are the horizontal velocities in $x$ and $y ; \eta$ is the height of the perturbed surface $(z=0) ; P_{\mathrm{a}}$ and $\left(\tau^{x}, \tau^{y}\right)$ are the atmospheric pressure fluctuation and the component of the wind stresses in $x$ and $y ; f$ is the Coriolis parameter (here assumed constant); $D$ is the depth of the mixed layer and $H$ is defined as the total depth of the ocean; $g$ is the acceleration of gravity; and $P_{\mathrm{m}}$ is the perturbed pressure at the mixed layer. The system is assumed to be in hydrostatic balance. $w_{\mathrm{S}}$ and $w_{\mathrm{B}}$ are the surface vertical velocity and the vertical velocity of the bottom of the mixed layer. (2.1) through (2.4) are derived assuming that the stresses vanish at the bottom of the mixed layer. For the interior layer,

$\frac{\partial u}{\partial t}-f v=-P_{x} / \rho_{0}$

$\frac{\partial v}{\partial t}+f u=-P_{y} / \rho_{0}$

$\frac{\partial w}{\partial t}=\frac{-P_{z}}{\rho_{0}}-\frac{g \rho^{\prime}}{\rho_{0}}$,

$\frac{\partial \rho^{\prime}}{\partial t}+w \tilde{\rho}_{z}=0$,

and

$\nabla_{H} \cdot \mathbf{V}=-w_{z}$.

The same definition holds for the variables in the interior. $\rho^{\prime}(x, y, z, t)$ is the density variation from the mean density $\bar{\rho}(z)$, and similarly for $P^{\prime}(x, y, z, t)$ where $\bar{P}(z)$ is in hydrostatic balance with $\bar{\rho}(z)$. It should be also noted that in the interior the hydrostatic assumption is not made, as it was in the mixed layer. The reason for doing this is that the hydrostatic approximation is well justified when the ratio of the vertical to the horizontal scale is very small $(h / L \ll 1)$. Our study will cover horizontal scales of the order of $10 \mathrm{~km}$. The depth of the mixed layer is of the order of $50 \mathrm{~m}$, and of the interior layer, $4000 \mathrm{~m}$. These numbers justify our approach concerning hydrostatic balance in the mixed layer.

In order to couple the systems of equations, we make use of these boundary conditions:

$w_{\mathrm{s}}=\frac{\mathrm{d} \eta}{\mathrm{d} t} \quad$ at $z=\eta$

$w=w_{\mathrm{B}} \quad$ and $\quad P=P_{\mathrm{m}} \quad$ at $z=-D ;$

and

$w=0 \quad$ at $z=-H$.

Taking the horizontal divergence of (2.1) and (2.2), multiplying by $D$, and making use of (2.4), we find

$$
\begin{aligned}
& -\left(w_{\mathrm{S}}-w_{\mathrm{B}}\right)_{t}=f D \zeta=-D g \nabla^{2} \eta-\frac{D \nabla^{2} P_{\mathrm{a}}}{\rho_{0}} \\
& +\frac{\nabla \cdot \tau}{\rho_{0}}
\end{aligned}
$$

where $\zeta$ is the vertical component of the vorticity in the mixed layer.

The curl of (2.1) and (2.2), using (2.4), is:

$D \zeta_{t}-f\left(w_{\mathrm{S}}-w_{\mathrm{B}}\right)=\frac{\nabla \times \tau}{\rho_{0}}$.

Combining the time derivative of (2.13) and (2.14) multiplied by $f$ and using (2.10) we derive an equation involving the vertical velocities and the forcing:

$$
\begin{gathered}
\left(w_{\mathrm{s}}-w_{\mathrm{B}}\right)_{t t}+f^{2}\left(w_{\mathrm{s}}-w_{\mathrm{B}}\right)-D g \nabla^{2} w_{\mathrm{S}} \\
=\left[D \nabla^{2} P_{\mathrm{a} t}-f \nabla \times \tau-(\nabla \cdot \tau)_{t}\right] / \rho_{0} .
\end{gathered}
$$

A similar derivation can be done for the interior layer. The curl of (2.5) and (2.6) gives the vertical component of the vorticity. Combining this with the divergence of (2.5) and (2.6), and differentiating in time, we obtain an equation relating the vertical 
velocity with the horizontal Laplacian of the time derivative of pressure:

$\left(w_{t t}+f^{2} w\right)_{z}=\nabla^{2} P_{t} / \rho_{0}$.

Then, by using (2.7), (2.8), and (2.9), we derive an equation involving only the vertical velocity $w$ :

$\left(w_{t t}+f^{2} w\right)_{z z}+\nabla^{2}\left(w_{t t}+N^{2} w\right)=0$,

where $N^{2}=-g \bar{\rho}_{z} / \rho_{0}$ is the square of the BruntVäisälä frequency.

Eq. (2.15) describes the dynamics of the mixed layer, and (2.17), the dynamics of the interior. The systems are coupled through boundary conditions (2.11), which, together with (2.16), give:

$\left(w_{t t}+f^{2} w\right)_{z}=g \nabla^{2} w_{\mathrm{s}}+\nabla^{2} P_{\mathrm{a}_{t}} \quad$ at $z=-D$.

(2.15) through (2.18) form a closed system that can be solved, given the expressions of $P_{\mathrm{a}}(x, y, t)$ and $\tau(x, y, t)$. Since previous papers deal only with wind stresses (Pollard, 1968, 1970) in the generation of low-frequency internal gravity waves, we will discuss, with a dimensional analysis, the role of both atmospheric pressure and wind stresses. The order of each forcing term in (2.15) can be expressed by

$\frac{D}{T L^{2}} P_{\mathrm{a}}, \frac{f|\tau|}{L}, \frac{|\tau|}{L T}$,

respectively, where $\left|P_{\mathrm{a}}\right|$ and $|\tau|$ are the characteristic amplitudes of the pressure fluctuations $\left(\left|P_{\mathrm{a}}\right|\right.$ $\left.=1 \mathrm{mb}=10^{2} \mathrm{~N} \mathrm{~m}^{-2}\right)$ and wind stresses $(|\tau|=0.1$ $\mathrm{N} \mathrm{m}^{-2}$ ). $D$, the depth of the mixed layer is about 50 $\mathrm{m}$; $L$ is a characteristic horizontal length; and $T$ is a characteristic time in which the forcing is applied. The difference between the second and third terms is due to the time scales. A comparison between $f$ and $T^{-1}$ for a time scale of $24 \mathrm{~h}$ shows that the second term is ten times larger than the third term, whereas a comparison for a 6 -h time scale shows that these terms only differ by a factor of two. The atmospheric pressure effect, however, involves a time scale as well as a horizontal length scale. This term becomes significant only for length scales on the order of $20 \mathrm{~km}$ and time scales less than $12 \mathrm{~h}$. In particular, for $L=20 \mathrm{~km}$ and $T=6 \mathrm{~h}$, the first term is approximately equal to the second term.

The next step will be to derive the governing equations for a system having constant stratification.

\subsection{Constant stratification}

A Fourier analysis in the horizontal plane allows us to rewrite eqs. (2.15), (2.17), and (2.18):

$$
\begin{gathered}
\left(\hat{w}_{\mathrm{s}}-\hat{w}_{\mathrm{B}}\right)_{t t}+f^{2}\left(\hat{w}_{\mathrm{s}}-\hat{w}_{\mathrm{B}}\right)+\left(k^{2}+l^{2}\right) D g \hat{w}_{\mathrm{S}} \\
=-\left[D\left(k^{2}+l^{2}\right) \hat{P}_{\mathrm{at}}-f \nabla \hat{x} \tau-\nabla^{\hat{*}} \tau_{t}\right] / \rho_{0}, \\
\left(\hat{w}_{t t}+f^{2} \hat{w}\right)_{z z}=\left(k^{2}+l^{2}\right)\left[N^{2} \hat{w}+\hat{w}_{t t}\right],
\end{gathered}
$$

and

$\left(\hat{w}_{t t}+f^{2} \hat{w}\right)_{z}=-\left(k^{2}+l^{2}\right)\left[g \hat{w}_{\mathrm{S}}+\frac{\hat{P}_{\mathrm{a} t}}{\rho_{0}}\right]$ at $z=-D$,

where the Fourier transform is defined

$\hat{f}=\frac{1}{2 \pi} \iint_{-\infty}^{\infty} f \mathrm{e}^{l(k x+l y)} \mathrm{d} x \mathrm{~d} y$.

We now define the Laplace transform of $g$ by $\bar{g}$

$\dot{g}=\int_{0}^{\infty} g \mathrm{e}^{-s t} \mathrm{~d} t$,

assuming that wind stresses and atmospheric pressure are $\tau=P_{\mathrm{a}}=0$ at $t=0$ and the perturbed velocities and acceleration are zero at $t=0$. The Laplace transforms of (2.20), (2.21), and (2.22) are:

$$
\begin{gathered}
\mathrm{s}^{2}\left(\hat{w}_{\mathrm{S}}-\hat{w}_{\mathrm{B}}\right)+f^{2}\left(\hat{\bar{w}}_{\mathrm{S}}-\hat{\bar{w}}_{\mathrm{B}}\right)+\left(k^{2}+l^{2}\right) D g \hat{w}_{\mathrm{S}} \\
=-s D\left(k^{2}+l^{2}\right) \frac{\hat{\hat{P}_{\mathrm{a}}}}{\rho_{0}}-\frac{f \overline{\nabla \times \tau}}{\rho_{0}}-\frac{s \overline{\nabla \cdot \tau}}{\rho_{0}}, \\
\left(s^{2}+f^{2}\right) \hat{w}_{z z}=\left(k^{2}+l^{2}\right)\left(N^{2}+s^{2}\right) \hat{w},
\end{gathered}
$$

and

$$
\begin{aligned}
& \left(s^{2}+f^{2}\right) \hat{w}_{z}=-\left(k^{2}+l^{2}\right) \\
& \quad \times\left[g \hat{w}_{\mathrm{s}}+s \frac{\hat{P}_{\mathrm{a}}}{\rho_{0}}\right] \text { at } z=-D,
\end{aligned}
$$

and the boundary condition at $z=-H$ is

$\hat{w}(-H)=0$.

The solution of (2.26) which satisfies the bottom boundary condition (2.28) is

$\hat{w}=w_{0} \sinh \gamma(z+H)$,

where

$\gamma=\left(k^{2}+l^{2}\right)^{1 / 2} \frac{\left(N^{2}+s^{2}\right)^{1 / 2}}{\left(s^{2}+f^{2}\right)^{1 / 2}}$. 
(2.27) gives the amplitude of $w_{0}$ as a function of $\hat{w}_{\mathrm{S}}$ and $\hat{P}_{\mathrm{a}}$; this relation is:

$w_{0}=-\frac{\left(k^{2}+l^{2}\right)\left[g \hat{\bar{w}}_{\mathrm{s}}+\frac{s}{\rho_{0}} \hat{\bar{P}}_{\mathrm{a}}\right]}{\left(s^{2}+f^{2}\right) \gamma \cosh \gamma(H-D)}$,

and using the boundary condition (2.11) at $z=-D$

$\hat{w}_{\mathrm{B}}=w_{0} \sinh \gamma(H-D)$.

Using (2.30) and (2.31) and (2.25), we derive a single equation for the surface vertical velocity $\hat{w}_{\mathrm{s}}$ :

$$
\begin{aligned}
& {\left[\left(s^{2}+f^{2}\right)+\left(\frac{k^{2}+l^{2}}{\gamma}\right) g \tanh \gamma(H-D)\right.} \\
& \left.+\left(k^{2}+l^{2}\right) g D\right] \hat{w}_{\mathrm{s}}=-\left(k^{2}+l^{2}\right) \frac{s}{\rho_{0}} \\
& \quad \times\left(\frac{\tanh \gamma(H-D)}{\gamma}+D\right) \hat{P}_{\mathrm{a}}-\frac{f \hat{\nabla \times \tau}}{\rho_{0}} \\
& \quad-\frac{s \bar{\nabla} \cdot \tau}{\rho_{0}},
\end{aligned}
$$

and $\hat{w}_{\mathrm{s}}$ will be:

$\hat{w}_{\mathrm{s}}=-\frac{F_{1}+F_{2}+F_{3}}{Q}$,

where $F_{1}, F_{2}$ and $F_{3}$ are the forcing terms defined as follows:

$F_{1}=\left(k^{2}+l^{2}\right) s\left[\frac{\tanh \gamma(H-D)}{\gamma}+D\right] \frac{\overrightarrow{P_{\mathrm{a}}}}{\rho_{0}}$,

$F_{2}=\frac{f \hat{\nabla} \times \tau}{\rho_{0}}$,

and

$F_{3}=\frac{s \bar{\nabla} \cdot \tau}{\rho_{0}}$.

$Q$ is defined as:

$Q=\left(s^{2}+f^{2}\right)+\left(k^{2}+l^{2}\right) g\left[\frac{\tanh \gamma(H-D)}{\gamma}+D\right]$.

Our main interest is to discuss the permanent features of the oceanic response; therefore, the expression for the surface vertical velocity given in (2.33) can be replaced by the surface height $\eta_{\mathrm{s}}$ :

$\hat{\bar{\eta}}_{\mathrm{s}}=-\left(F_{1}+F_{2}+F_{3}\right) / s Q$.

Since we assume that $w_{\mathrm{s}}=\partial \eta_{\mathrm{s}} / \partial t$, the steady-state response to these complicated forcings can be evaluated by simply using the property of the Laplace transform which is that a function at a very long time is equal to the contribution of the residue at $s=0$. All of the contributions for the zeroes of $Q$ are the response of internal inertiagravity waves. Inspecting the three atmospheric forcing terms from (2.34), it can be concluded that only the curl of the stress will produce a steadystate response in the ocean, as long as none of the three forcing terms has a pole at $s=0$. Then, the Fourier component of the inverse Laplace transform for the surface height is given by

$$
\hat{\eta}_{\mathrm{s}}=-\frac{1}{2 \pi} \frac{\frac{1}{\rho_{0}} f \hat{\nabla}_{\tau_{(s=0)}}}{f^{2}+\left(k^{2}+l^{2}\right) g\left[\frac{\tanh \gamma_{0}(H-D)}{\gamma_{0}}+D\right]}
$$

where $\gamma_{0}=N\left(k^{2}+l^{2}\right)^{1 / 2} / f$. Similarly, the interface height at the bottom of the mixed layer can be calculated by using (2.30), (2.31) and (2.37),

$\hat{\eta}_{\mathrm{B}}=-\frac{\left(k^{2}+l^{2}\right)^{1 / 2}}{f N} g \tanh \gamma_{0}(H-D) \hat{\eta}_{\mathrm{s}}$

As before, we assume that $w_{\mathrm{B}}=\partial \eta_{\mathrm{B}} / \partial t$. The Fourier transform used to find $\eta_{\mathrm{s}}$ and $\eta_{\mathrm{B}}$ will be given by the sum of all the residues, i.e., from the forcing term, the curl of $\tau(s=0)$, and from the internal impedance

$\left(Q=f^{2}+\left(k^{2}+l^{2}\right) g\left[\frac{\tanh \gamma(H-D)}{\gamma}+D\right]\right)$.

The point we would like to stress here is the role that the stratification plays in the response; it only affects the zeroes of $Q$ which will be the poles that contribute to the Fourier transform of (2.37) and (2.38). We can easily see that for small wavenumbers $\left(\gamma_{0}(H-D)<1\right), Q$ simply reduces to $f^{2}+\left(k^{2}\right.$ $\left.+l^{2}\right) g H$; which is independent of the stratification and gives the well-known barotropic mode as a response. The role of the stratification can be seen more clearly if we assume that $D$ is negligible 
compared with $H$ and then the solutions for $Q=0$ will be close to $\gamma_{0} H \approx i n \pi$. Using the definition of $\gamma_{0},\left[\left(k^{2}+l^{2}\right)^{1 / 2}= \pm \operatorname{in}(\pi f / N H)\right]$ we find that $N H / f n$ is the $n$th internal Rossby radius of deformation. Certainly the scale depends on the stratification, but since the numerator of $(2.38)$ (the response at the bottom of the mixed layer) also has a $\sinh \gamma_{0} H$ which will go to zero for the same values of $\gamma_{0} H$, we find that all of the internal modes are very inefficient in making a significant contribution to the surface forcing. This is in agreement with previous results (Bolin, 1953; Pollard, 1972), which found that higher internal modes play a secondary role in the adjustment problem; nevertheless, the previous results did not clarify the exact role of the stratification. $\eta_{\mathrm{B}}$ is equal to the forcing for any horizontal scale, even for scales smaller than the barotropic Rossby radius of deformation. It does not have a preferential scale related to any of the baroclinic Rossby radius of deformation scales. In fact, (2.38) shows that the response cannot be expressed by only the contribution of all the internal modes, and this is perhaps the most important conclusion of this result. Physically as well as mathematically it becomes evident that all the internal modes produce zero contribution at the surface, whereas the response to atmospheric forcing is certainly not zero. To proceed with the discussion of what the final steady state response is, and what the differences are between the stratified and non-stratified cases, we must keep in mind that the only forcing that will contribute to the steady-state response is the curl of $\tau$ evaluated at $s$ $=0$, regardless of the time evolution of $\tau$. Therefore, the basic equations previously derived in (2.15) and (2.17), and the boundary condition (2.18) can be simplified if the independent variable is the isopycnic height $\eta_{\mathrm{I}}$ where $\eta_{\mathrm{S}}$ and $\eta_{\mathrm{B}}$ are defined to be the values of $\eta_{1}$ at $z=0$ and $X=-D$, respectively; this being in agreement with the solution of (2.37), the basic equations (2.15), (2.17) and (2.18) become:

$$
\begin{aligned}
& \left(\eta_{\mathrm{B}}-\eta_{\mathrm{s}}\right) f^{2}+g D \nabla^{2} \eta_{\mathrm{s}}=\frac{1}{\rho_{0}} \frac{f}{2 \pi} \\
& \times{\overline{\nabla \times \tau_{(s=0)}}} \text { for }-D<z \leqslant 0, f^{2} \eta_{\mathrm{i}_{z z}}+N^{2} \nabla^{2} \eta_{1} \\
& =0 \text { for }-H \leqslant z \leqslant-D \text {, } \\
& \text { and } \\
& \left.\begin{array}{l}
\eta_{\mathrm{I}}=\eta_{\mathrm{B}} \\
\eta_{\mathrm{s}}=\frac{\mathrm{g}}{f^{2}} \nabla^{2} \eta_{\mathrm{S}}
\end{array}\right\} \quad \text { at } z=-D \text {. }
\end{aligned}
$$

Actually, the first equation is nothing more than the conservation of potential vorticity in the mixed layer; the second equation is for stratified flow and is zero because no forcing is present. The two systems are connected by the boundary conditions expressed in the last two equations.

\section{The response}

In this section we shall first evaluate the stresses that affect the steady response and look at the horizontal and vertical structures.

\subsection{Steady contribution from transient stress}

Without losing generality we might choose the wind stress to be a separate function of space and time $\left[\tau(x, y, t)=\tau_{0} T(t) \bar{G}(x, y)\right]$, where $\tau_{0}$ is a dimensional constant. Now the direct evaluation of the Laplace transform of $T$ at $s \rightarrow 0$ can be done. Let us say then that the storm in question, assumed for the moment to be a front, is generated and decays in the same place in a matter of a few days. For simplicity, $T$ can be written as

$T(t)=[\tanh a t-\tanh \beta(\mathrm{t}-\Delta t) H(t-\Delta t)]$,

where $\alpha$ and $\beta$ are the generation and decay rates, respectively, $\Delta t$ is the duration time of the storm, and $H$ is the Heaviside function. Fig. 1 shows the shape of $T(t)$ vs time. By choosing the parameters properly, this function can be like a step function between 0 and $t$, or can represent a constant wind stress if $t$ is infinite. This function allows us to determine the response for specific time intervals during the storm. Also, note that our assumption for the separation of time and space does not allow for a more realistic case, namely, a storm moving for a period of time and then decaying over the

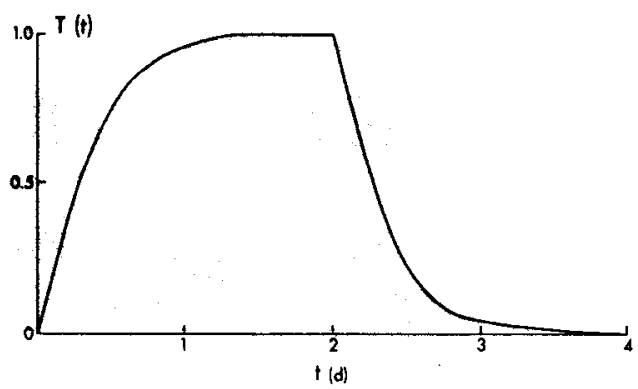

Fig. 1. The non-dimensional wind stress function $T$ as a function of time. 
ocean, which is more characteristic of frontal behavior. The latter case, however, will be discussed when numerical simulations are presented. The present forcing, although simplified, captures the important elements of the most realistic case. Let us then examine the Laplace transform of $\bar{T}(t)$, which by using known properties of the transform can be written as:

$$
\begin{array}{r}
\bar{T}(t)=\frac{1}{\alpha}\left[\frac{1}{2} \psi\left(\frac{1}{4} \frac{s}{\alpha}+\frac{1}{2}\right)-\frac{1}{2} \psi\left(\frac{1}{4} \frac{s}{\alpha}\right)-\frac{a}{s}\right] \\
-\frac{a \mathrm{e}^{\Delta t s}}{\beta}\left[\frac{1}{2} \psi\left(\frac{1}{4} \frac{s}{\beta}+\frac{1}{2}\right)-\frac{1}{2} \psi\left(\frac{1}{4} \frac{s}{\beta}\right)-\frac{\beta}{s}\right],
\end{array}
$$

where $\psi(z)$ is the logarithmic derivative of the gamma function and has the following properties:

$$
\psi(1+z)=\psi(z)+\frac{1}{2}, \quad \psi(z \rightarrow 0)=\psi(1)-\frac{1}{2},
$$

$\psi(1)=-\gamma, \quad \psi\left(\frac{1}{2}\right)=-\gamma-2 \ln 2$,

Then its limit for $s=0$ can be easily calculated:

$K_{0}=\left.\bar{T}(t)\right|_{s=0}=-\left(\frac{1}{a}-\frac{1}{\beta}\right) \ln 2+\Delta t$.

The simple forcing amplitude from this rather complicated transient stress can be physically explained in the following manner. The first term reflects the difference between the growth and decay time of the storm. If the storm evolves in a symmetric manner, the contribution to the response will be zero, because there is an exact balance between the growth and decay terms. The second term is directly proportional to the duration of the storm, $\Delta t$. It is well-known that the oceanic response grows linearly in time for a steady stress, and since $\Delta t$ is the time in which the stress is constant, the response will take that long to grow. It is interesting to note that the permanent oceanic response is given only by the amplitude, $\bar{T}(t)_{s=0}$. The rest of the transience of the wind stresses goes into the wave energy, and none of this energy is converted to the geostrophic mode, at least by linear processes.

Next let us look at the actual spatial shape of the response to the given forcings.

\subsection{Response for the homogeneous ocean, $N^{2}=0$}

To properly understand the effect of this stratification, let us first discuss the response in a homogeneous solution obtained by setting $N=0$ and by letting $D$ go to $H$, the total depth of the ocean. If $\eta^{\prime}$ is the non-dimensional height, $\eta / H$, and the horizontal variables $X$ and $Y$ are nondimensionalized by $L$, the length of the storm, then the system described by (2.39) reduces to the wellknown equation,

$\nabla^{2} \eta^{\prime}-\eta^{\prime}\left(L^{2} / R B^{2}\right)=G_{0} \nabla X \bar{G}(X, Y)$,

where $R B$ is the barotropic Rossby radius of deformation, $R B=(g H)^{1 / 2} / f$, and $G_{0}\left(\tau_{0} K_{0} L /\right.$ $\left(2 \pi \rho_{0} H f R B^{2}\right)$ is the non-dimensional amplitude of the stresses. Out next step then is to evaluate the right-hand side of (3.4) from a given transient forcing. This problem has been discussed to a great extent in the period of time from Rossby (1937) to the present, but perhaps the emphasis was on the initial-value problem rather than the boundaryvalue problem stated here. Nevertheless, even in the former case, the work by Veronis and Stommel (1956), Pollard (1970), Geisler (1970), and Blumen (1972) has more or less clarified different aspects of the geostrophic adjustment due to external forcing. Yet, we believe that in order to clarify the discussion in the following sections, we should first review some of the characteristics of the response to a localized forcing in this particular case. Let us then assume for simplicity that $\bar{G}$ is given by

$\bar{G}(X)=(0,1 / \cosh (X))$.

Since only the $y$-component of $G$ will be relevant for the calculation of the stresses in (3.4), let us call this $G(X)$. The curl of this stress $\bar{G}$, which is the forcing of (3.4), is equal to

$G_{x}(X)=-\sinh (X) / \cosh ^{2}(X)$.

We can directly integrate (3.4) with this forcing, and the solution can be seen in Fig. 2, where the non-dimensionalized forcing function $G_{x}$ the surface height $\eta^{\prime}=\eta / H$, and the geostrophic velocity, $V_{N}=(V / f R B)$ are shown for four different ratios between the storm scale, $L$, and the barotropic Rossby radius of deformation, $R B$. We can see that $\eta^{\prime}$ and $G_{x}$ have similar scales when $L / R B>1$. For ratios less than 1 , the surface height decays much slower than the forcing, and the decay rate outside the region of forcing is $R B$. The scale of the largest slope or the scale of the jet is equal to that of the localized forcing regardless of the ratio. In fact, it can be shown that $V$ is approximately equal to $\tau_{x x}$ This result is not in contradiction with previous 

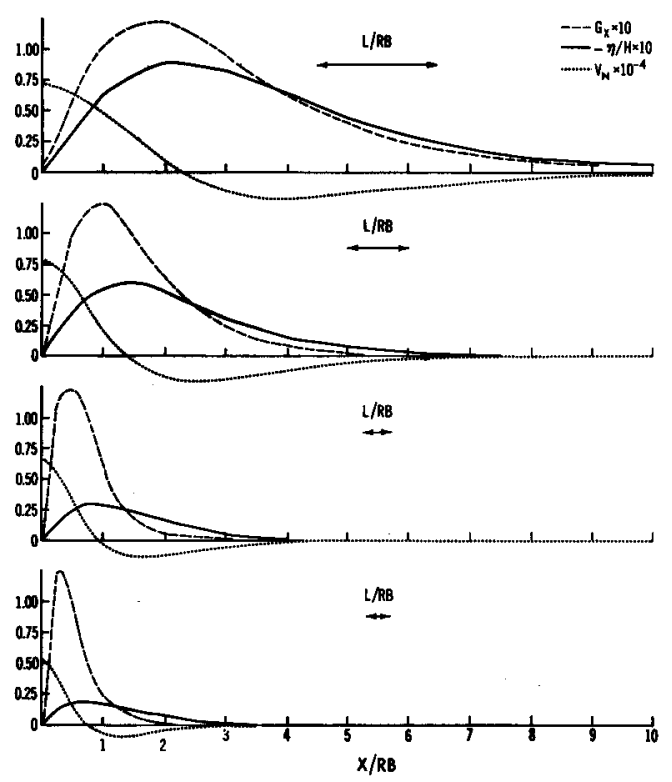

Fig. 2. The non-dimensionalized forcing function $G_{x}$, surface height $\eta / H$, and geostrophic velocity $V_{\mathrm{N}}$ as a function of a non-dimensional length, $X / R B$. These curves are shown for decreasing values of the nondimensional aspect ratio $L / R B$.

works. In this simplified problem the ratio $L / R B$ does not have any selective properties. However, one should note that a slight maximum occurs in the $v$ velocity, Fig. 2 , for the ratio $L / R B=1 . L / R B$ only determines the ratio of potential to kinetic energy in geostrophic flow, as is well known. Since the geostrophic velocity is $V=(g / f)(\Delta \eta / L)$, the kinetic energy is given by $K=\frac{1}{2} \rho H g^{2}\left(\Delta \eta^{2} / f^{2} L^{2}\right)$; and in that the potential energy is equal to $P=\frac{1}{2}$ $\rho g \Delta \eta^{2}$, the ratio, $K / P$ is equal to $(R B / L)^{2}$. This fact gives a simple explanation of geostrophic adjustment. Given an initial perturbation of the surface height, which is equivalent to an input of potential energy, storms with scales larger than $R B$ will quickly adjust to geostrophic flow, because as we have seen in that limit the ratio between kinetic and potential energy is very small $(P \gg K)$. Only a small adjustment in the initial potential energy is required to produce the small amount of geostrophic kinetic energy for its final balance where the final geostrophic potential energy is nearly equal to the initial input energy. On the other hand, since the ratio $K / P \gg 1$ for storms with scales smaller than $R B$, the final geostrophic potential energy would be much smaller than the initial input of potential energy $K \gg P$ resulting in a big adjustment from the initial conditions and a resulting low level of geostrophic energy. Conversely, the initial input of momentum or equivalent kinetic energy will give the opposite result. In our example, however, the amount of energy going to the geostrophic system has already been shown to be proportional to $\Delta t^{2}$, regardless of the ratio $L / R B$. The partition of potential and kinetic energy in this geostrophic flow is still proportional to $(L / R B)$. We should point out that the response is highly sensitive to the time history of the forcing, and that the response to initial-value problems as stated in the past can be misleading. We will return to this point when a more sophisticated system than the present simple barotropic flow will be treated.

\subsection{The stratified case $N^{2} \neq 0$}

To discuss the effect of stratification let us return to the system described by (2.39), and let us non-dimensionalize it as follows:

$z=H z^{\prime}, \quad \eta=H \eta^{\prime}, \quad D=\delta H, \quad x=R N x^{\prime}$,

where

$R N=N_{0} H / f$,

and $R N$ is the baroclinic Rossby radius of deformation. Then, dropping the primes of the variables, the system (2.39) becomes:

$\left(\eta_{\mathrm{B}}-\eta_{\mathrm{S}}\right)+\delta\left(\frac{R B}{R N}\right)^{2} \eta_{\mathrm{s}_{x x}}=G_{0} G_{x}$,
$\eta_{\mathrm{Igz}_{z}}+\eta_{\mathrm{Lxx}}=0$ for $-1 \leqslant z \leqslant-\delta$,

and

$\left.\begin{array}{l}\eta_{\mathrm{I}}=\eta_{\mathrm{B}} \\ \eta_{\mathrm{I}_{z}}=\left(\frac{R B}{R N}\right)^{2} \eta_{\mathrm{S}_{x x}}\end{array}\right\} z=-\delta$.

Note that $\eta_{\mathrm{S}}$ and $\eta_{\mathrm{B}}$ are now the non-dimensional heights at the surface and interface, respectively; $\eta_{1}$ is the height of the isopycnics and is a function of height. The forcing was assumed to be only a function of $x . G_{x}$, as before, is the non-dimensional form of the curl of $\tau$, with $G_{0}$, the non-dimensional amplitude, defined as $\left(\tau_{0} K_{0}\right) /\left(2 \pi \rho_{0} R N H f\right)$.

The role of stratification in the oceanic response can be analyzed by using the simple example of the solution of the system (3.7) and some assumptions that we will relax later. First, since $R B \gg R N(2000$ 
$\mathrm{km}$ for $R B$ in the real ocean whereas $R N$ is no more than $100 \mathrm{~km}$ ), and since our main interest is to discuss storms with length scales on the order of $R N$ or smaller $(100 \mathrm{~km})$, we can see that $\eta_{\mathrm{s}}$ becomes much smaller than $\eta_{\mathrm{B}}$ (using the boundary condition for (3.7)). Under this assumption the first equation of (3.7) reduces to

$\eta_{\mathrm{B}}+\delta \eta_{\mathrm{I}_{2}}(-\delta)=G_{0} G_{x}$.

Furthermore, for the sake of simplicity, the non-dimensional function $\eta$ and its derivative are of the same order $\left(\eta_{z} \approx \eta_{\mathrm{B}}\right)$ and since $\delta \ll 1$, the system (3.7) and condition (3.8) could further be approximated to

$\eta_{L_{z}}+\eta_{L_{\mathrm{hx}}}=0$ for $-1 \leqslant z \lesssim 0$,

with the boundary condition,

$\eta_{\mathrm{I}}(-\delta \approx 0)=\eta_{\mathrm{B}}=G_{0} G_{x}$

Let us assume that the forcing, $G_{x}(x)$, is given by:

$G_{x}(x)=\mathrm{e}^{-\left(x^{2} / L^{2}\right)} \sin \left(2 a x / L^{2}\right)$,

where $a$ and $L$ are two parameters which control the horizontal scales of wind stress. The transform is then

$G_{x}(k)=P \mathrm{e}^{-1 / 4(L / R N)^{2} k^{2}} \sinh (a k / R N)$

where

$P=\mathrm{e}^{-(a / L)^{2} /(L \pi / R N) .}$

It follows then from (3.11) that the scale of the forcing function can be changed only by changing $L$. That being so and without losing generality $a$ can be set equal to $R N$. Substituting (3.12) in the expression for $\eta_{1}(k)$ that is assumed to be of the form $\eta_{0} \sinh k(1+z)$ to satisfy (3.9):

$\hat{\eta}_{\mathrm{I}}(k)=G_{0} P \mathrm{e}^{-1 / 4(L / R N)^{2} k^{2}} \sinh k(1+z)$,

which for $z=0$ is the same as the forcing $G_{x}(k)$. Seeing the analogy between this and (3.12), the inverse transform becomes trivial, and $\eta_{1}(x, z)$ is given by:

$\eta_{1}(x, z)=G_{0} \mathrm{e}^{(R N / L)\left[(1+z)-1-x^{2}\right]}$

$$
x \sin \left[2[R N / L)^{2}(1+z) x\right],
$$

which satisfies the bottom boundary condition $\eta_{1}(x,-1)=0$. The non-dimensional response for different values of the aspect ratio of the storm length to the Rossby radius of deformation is shown in Fig. 3, where the amplitude of $\eta_{\mathrm{I}}$ at two

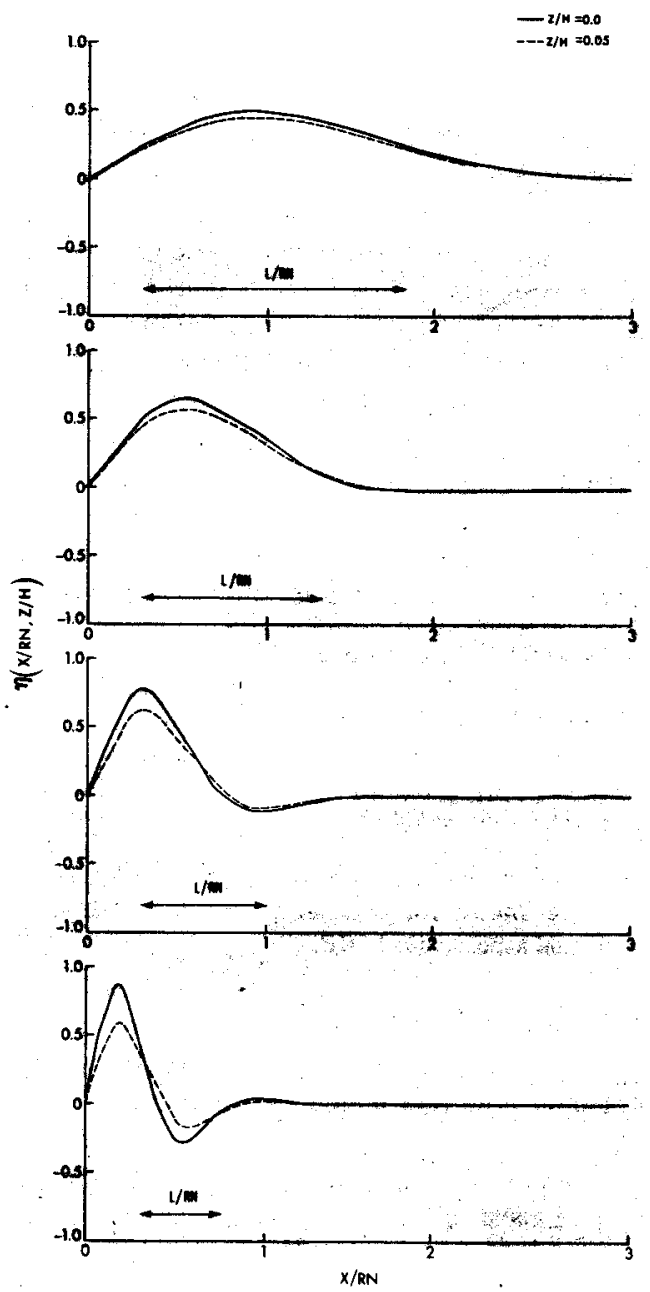

Fig. 3. The non-dimensional response, $\eta_{1}$, (for $z=0$, and $z=-0.05$ ) in relation to four different aspect ratios of the storm scale to the baroclinic Rossby radius of deformation, $L / R N$.

different depths is shown (the height at the bottom of the mixed layer $(z=0)$ and $z=-0.05$ which, for the dimensional system, will correspond to a depth of $200 \mathrm{~m}$ ). Again we see that the horizontal scale is in the order of the forcing for all ratios, and the depth of the penetration is a function of the aspect ratio, $L / R N$, which confirms what we had stated in the previous discussion.

\subsection{Finite mixed layer}

We recognize the fact that in order to obtain (3.15), we had assumed an infinitesimally small mixed layer and a storm scale smaller than the 
barotropic Rossby radius of deformation $(R B \approx$ $1000 \mathrm{~km})$. One should also realize, however, that the solution of (3.15) is a complete solution of the interior system from $0 \geq z \geq 1$ and satisfies all the boundary conditions. What is left to determine is the forcing function $G$, and a surface height that for a given finite mixed layer will satisfy the first equation in (3.7). For illustrative purposes we can do the reverse problem of satisfying the relation of $\eta_{\mathrm{I}}$ and $\eta_{\mathrm{s}}$ at $z=0$ given in the system of (3.7); then, substituting $\eta_{\mathrm{I}}(x, 0)$ and $\eta_{\mathrm{s}}$ in the surface equation we can calculate the wind stress forcing $G_{x}$. Since the full solution of the numerical model will be presented in the sections to follow, we shall not elaborate on that topic here. Notice that, however,

$\eta_{\mathrm{I} z}=(R B / R N)^{2} \eta_{\mathrm{S} x x} \quad$ at $z=-\delta$.

With the boundary condition of (3.7) and using the expression of $\eta_{1}$ from (3.15), one can actually calculate $\eta_{\mathrm{s}}$; but, since the expression is not simple to integrate, it will be convenient to first estimate the terms that appear in (3.7). Let us calculate $\eta_{s_{x}}$, which is rather simple to integrate and is equal to:

$$
\begin{aligned}
& \eta_{\mathrm{s}_{x}}=(R N / R B)^{2} \int \eta_{\mathrm{I}_{\mathrm{z}}} \mathrm{d} x=-(R N / R B)^{2} G_{0} \\
& \quad \times \cos \left(2(R N / L)^{2} x\right) \mathrm{e}^{-(R N / L)^{2} x^{2}} .
\end{aligned}
$$

Notice that to simplify (3.17), and without any major consequence, $\eta_{\mathbf{I}_{z}}$ was evaluated at $z=0$ rather than at $-\delta$. Since the dependence of $\eta_{\mathrm{I}_{z}}$ (3.15) is through the term $1+z$, the approximation implies to change $1-\delta$ for unity. The maximum value for $\eta_{\mathrm{S}_{x}}$ is given at $x=0$ by

$\eta_{\mathrm{s}_{x_{\max }}}=-G_{0}(R N / R B)^{2}$.

We can compare this with the maximum slope of $\eta_{\mathrm{I}}$ from (3.15) which is:

$\eta_{\mathrm{Lx}_{\max }}=G_{0} 2(R N / L)^{2}$.

Now, if the ratio of these two quantities is assumed to be proportional to the ratio of their amplitudes, we have

$\left|\frac{\Delta \eta_{\mathrm{s}}}{\Delta \eta_{\mathrm{I}}}\right| \approx \mathrm{O}\left((L / R B)^{2}\right)$.

This ratio then indicates that the interface slope at the bottom of the mixed layer is always much larger than the free-surface height slope when the storm scale, $L$, is smaller than the barotropic Rossby radius of deformation. This result is in full agreement with the well known rigid-lid approxi- mation. This conclusion does not imply that the velocity in geostrophic balance with the freesurface height is smaller than the geostrophic velocity in balance with the height of the interface. Recall that the steady-state solution relates the vertical shear, $V_{z}$, to the internal height, $\eta_{1}$, as:

$V_{z}=-\eta_{\mathrm{I}_{z}}(0) N^{2} / f$

Then differentiating (3.15) at the maximum shear $(x=0)$, and non-dimensionalizing the velocity by $V / R N f$, the non-dimensional maximum shear is given by

$V_{z}(0, z)=-G_{0} 2(R N / L)^{2}(1+z) \times \mathrm{e}^{(R N / L)^{2}\left[(1+z)^{2}-1\right]}$,

from which we can obtain $V(0, z)$ by directly integrating (3.22):

$V(0, z)=-G_{o} \mathrm{e}^{(R N / L)^{2}\left[(1+z)^{2}-1\right]}+V_{0}$.

This geostrophic velocity evaluated at its maximum value is

$V(0,0)=-G_{0}+V_{0}$.

Recalling that the non-dimensional geostrophic relation for $V_{\mathrm{s}}$ is:

$V_{\mathrm{S}}(0)=(R B / R N)^{2} \eta_{\mathrm{S}_{x}}$,

and substituting this expression in (3.18), we see that $V_{s}(0)=V_{\max }(0,0)$ if the constant of integration is set to zero. As to the question of recalculating the stresses that produce $\eta_{\mathrm{I}}$ in (3.15), we can approximate (3.7) by assuming $\eta_{\mathrm{s}} \ll \eta_{1}$, and then we can write

$\eta_{1}(0)+\delta(R B / R N)^{2} \eta_{\mathrm{S}_{x x}}(x)=G_{0} g_{x}(x)$.

If we substitute the value $(R N / R B)^{2} \eta_{\mathrm{I}_{z}}$ for $\eta_{\mathrm{S}_{x x}}$ in (3.26), the full forcing term compatible with a mixed layer and $\eta_{1}$ is:

$g_{x}=\eta_{1}(x, 0)+\delta \eta_{\mathbf{L}_{z}}(x, 0)$

where $g_{x}$ is the forcing term of the complete solution; we should keep in mind that $G_{x}$ was the forcing term that approximately gave $\eta_{\mathrm{l}}$. As a matter of fact, the only difference between $g_{x}$ and $G_{x}$ is the effect of the mixed layer which appears in the second term on the right-hand side of (3.27). This last term is of the order of $\delta(R N / L)^{2}$ and will be negligible compared with the first term insofar as $\delta(R N / L)^{2} \ll 1$. Calculating $R N$ using an average stratification in the ocean, $4 \times 10^{-3} \mathrm{~s}^{-1}$, which is perhaps a little large, gives a value of $160 \mathrm{~km}$. Since 
the mixed layer is no more than $50 \mathrm{~m}$ in depth, $\delta$ then has a value of about $10^{-2}$. Any storm scale, $L$, larger than $16 \mathrm{~km}$ will make this term negligible. We shall return to this point, however, when we discuss the penetration depth.

\subsection{Depth of penetration}

It seems apparent from the previous discussion that the stratification has no role in selecting the horizontal response scale when surface forcing is considered. The only scale selection should result through the internal Rossby radius of deformation which has been shown to have nil effect in the response of the interface height (2.38). Since the governing equation for the response in the interior, $\eta_{z z}+\eta_{x x}=0$, implies that the vertical nondimensional scale, $h$, should be equal to $l$, the horizontal non-dimensional scale, or $h / H=L f /$ $N H$. If the horizontal scale is determined by the storm, its aspect ratio $(h / l)$ will be given by $f / N$. We shall use the simple example previously discussed to determine the penetration depth for a given scale, $L$. This is an important question that has not been properly addressed in previous papers on geostrophic adjustment. This was mainly because the examples given considered either homogeneous oceans or large-scale storms. In this case, since our main interest is to address mesoscale forcing, the penetration depth, which is smaller than the depth of the ocean, becomes highly important. The way in which we define the penetration depth is arbitrary. Let us define it as the depth at which the surface velocity decays by a factor $e$. From (3.23),

$$
\begin{aligned}
& V\left(0, h_{e}\right)=-G_{0} \mathrm{e}^{(R N / L)^{2}\left[\left(1-h_{e}\right)^{2}-1\right]} \\
& =\mathrm{e}^{-1} V(0,0) .
\end{aligned}
$$

Remembering that $V(0,0)=-G_{0}$, then $h_{e}$ becomes

$$
h_{e}=1-\left(1-(L / R N)^{2}\right)^{1 / 2} \text {. }
$$

Notice that this penetration scale involves a barotropic component that is contained in (3.23). It would be convenient, however, to have a purely baroclinic scale, and this can be defined as a ratio of a higher derivative of $z$. If $h_{e_{\mathrm{b}}}$ (baroclinic) is defined as $V_{z} / V_{z z}$

$$
h_{e_{\mathrm{b}}}=1 /\left(1+2(R N / L)^{2}\right) \text {. }
$$

These two scales of penetration are shown in Fig. 4. For aspect ratios larger than or equal to one, $h_{e}$ is equal to the total depth of the ocean (barotropic

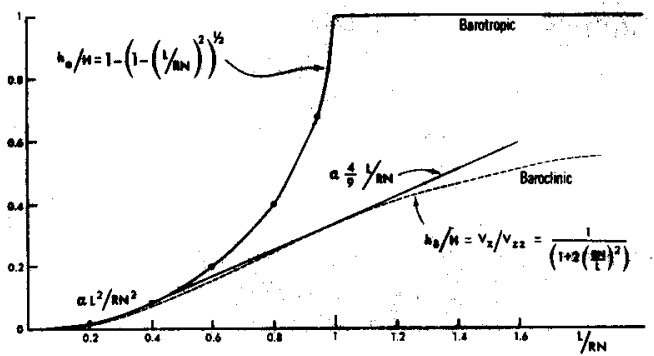

Fig. 4. The non-dimensional barotropic (3.29) and baroclinic (3.30) penetration scales as a function of the aspect ratio $L / R N$.

response), whereas for very small aspect ratios, the penetration is $\frac{1}{2}(L / R N)^{2} H$. For example, given the values previously used for a typical ocean, for $R N$ $=160 \mathrm{~km}$, and for storms of the order of $80 \mathrm{~km}$, the depth of penetration is on the order of $500 \mathrm{~m}$. Notice that the baroclinic scale height is linearly dependent on $L / R N$ for values of $L / R N$ close to 1 .

In conclusion, let us say that most of the realistic examples give penetration depths of $100 \mathrm{~m}$ or deeper, with mixing depths on the order of several tens of meters. The role of a variable stratification and more realistic storm forcings are considerably more difficult to treat in the present framework; therefore, numerical solutions for different and more realistic conditions will be presented in the next section.

\section{The numerical solution}

Previous studies (Orlanski and Ross, 1977; Ross and Orlanski, 1977) on the evolution of atmospheric fronts using two-dimensional numerical models were successful in describing the role of the different cross-stream circulations of the front in maintaining sharp surface temperature gradients for considerably long periods of time. In addition, the model solution was also able to show the role of frontal lifting in producing deep convection such as the frontal squall line. This same basic model can be converted to an ocean model forced by surface conditions produced by a numerically simulated atmospheric front. In this case, a front will exert forcing through wind stresses and atmospheric pressure as well as through temperature contrast. The ocean model, as in the atmospheric case, uses a turbulent eddy viscosity parameterization which is a function of the local Richardson number, and it 

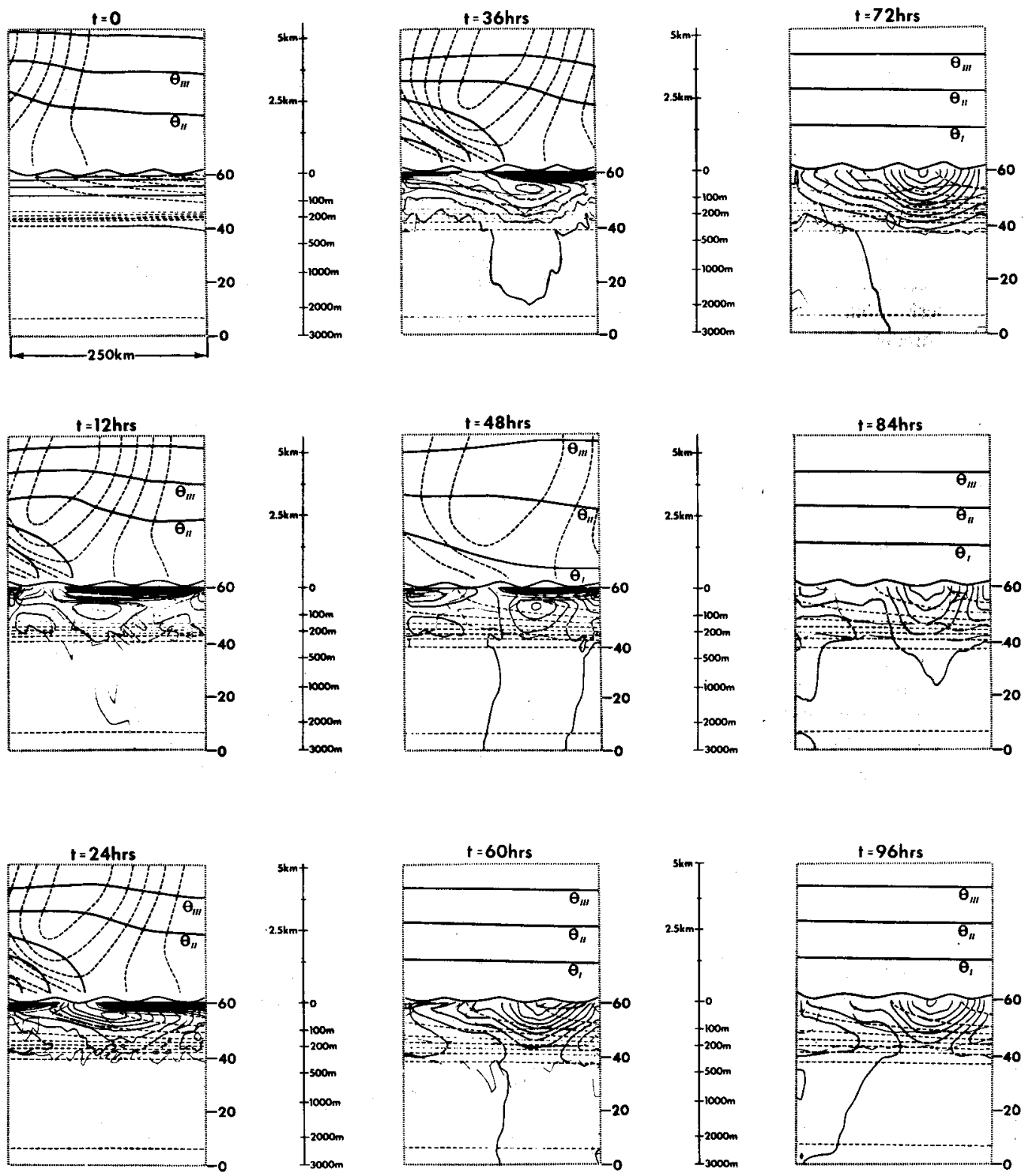

Fig. 5. A typical time sequence of the model showing $x-z$ cross sections of the oceanic response as an atmospheric front moves through the domain at $1 \mathrm{~m} \mathrm{~s}^{-1}$. The front is in the domain $(200 \mathrm{~km})$ for $50 \mathrm{~h}$ with the duration of the decay lasting $10 \mathrm{~h}$. Contours of potential temperature (heavy solid lines) and $v$ velocity (heavy dashed lines) are shown for the atmospheric front. Contours of the $v$ current and constant-density surfaces are denoted by the light solid and light dashed lines respectively. The air-sea boundary is represented by the undulating surface.

can produce its own boundary layer without prescribing the depth of the mixed layer as was previously done. For completeness, however, we will discuss the solutions for different prescribed turbulent eddy viscosity profiles to determine the effect of the turbulence profile on the oceanic response. The actual details of the numerical setup and characteristics of the model are described in 
the Appendix. We shall therefore focus our attention on discussing the results of these numerical solutions in order to extend the range of our previous conclusions. With that in mind, our discussion will encompass the behaviour of the transient response, the penetration depth dependence on different stratifications, the characteristics of the responses to four different forcing scales, and the dependence of the response on the eddy viscosity profile. The results presented in this section were obtained from a linearized version of the full model (Appendix); experiments which include the non-linear effects in a stratified ocean will be presented in Section 5. Different numerical experiments along with some of their most significant features are shown in Table $\mathrm{A} 1$ of the Appendix.

Fig. 5 is a graphic illustration of the model in a time sequence (12-h intervals) showing the atmospheric front and the oceanic response. Specifically, this solution was done with the full non-linear equations, a non-linear turbulence parameterization (a function of the local Richardson number), and a realistic ocean stratification. The atmospheric solution was obtained from Orlanski and Ross (1977). The important point here is to illustrate what to expect and to note that we only calculate the oceanic flow in this study. Note that after $36 \mathrm{~h}$ the maximum surface winds are in the middle of the domain, and the ocean has reacted with a very intense surface current confined to the upper layers $(60 \mathrm{~m})$ and a much weaker current located at about $100 \mathrm{~m}$. In order to appreciate the scales involved here, we have included vertical grid numbers on the right side of each cross-section and a height scale between the cross-sections. The atmospheric front decays with a time scale of half a day, and we can see that it has weakened considerably after $48 \mathrm{~h}$ and has totally dissipated after $60 \mathrm{~h}$. On the other hand, in the ocean we see that the Ekman flow with its intensely packed contours decays slowly to a state in which only the baroclinic response appears and remains in a steady state balance with the horizontal density gradients for the remainder of the integration. At this point it would be appropriate to discuss the transient behaviour of the numerical solution, since it is generally applicable to all the cases we shall discuss; then, we shall present specific descriptions of the spatial characteristics of the atmospheric forcing and ocean response.

\subsection{Transient behaviour}

In Fig. 6 the time histories of $v$ and $u$ (upper and lower respectively) are shown for various depths (surface to $1000 \mathrm{~m}$ ) for a case similar to that shown in Fig. 5. The profile is taken at $150 \mathrm{~km}$ from the left boundary of the domain, keeping in mind that the total length of the domain is $250 \mathrm{~km}$ in this case. Perhaps the most prominent feature here is that the surface currents respond abruptly to the frontal movement. In examining this response, there are three phases that should be recognized. Initially, the front moves with a constant speed of 1 $\mathrm{m} \mathrm{s}^{-1}$ for the first $24 \mathrm{~h}$, after which frontolysis occurs for the next $26 \mathrm{~h}$. Finally, after $50 \mathrm{~h}$ the forcing is non-existent. As one would expect, the ocean current seems to respond by building an Ekman flow with large inertial waves having a response time scale of 1 pendulum day. Notice the linear growth of the responses in the lower levels in the $v$ component and the lack of it in the $u$ component. After $60 \mathrm{~h}$, a steady response of $0.12-0.15 \mathrm{~m} \mathrm{~s}^{-1}$ can be seen superimposed on a field of inertial-gravity waves in the flow perpendicular to the plane and only inertial-gravity waves in the $u$ component. At that time the permanent response has attained a geostrophic balance.

It should be remembered that these results agree very well with the conclusions drawn in (3.3), that the amplitude of the geostrophic response is basically proportional to the time in which the forcing is applied $(\Delta t)$. It is easy to infer from the results of Fig. 6 that if the forcing had been dissipated in half the time, the geostrophic response would be half as intense.

In summary then, it seems plausible to think that fronts which become stationary over the ocean prior to decaying could transfer enough momentum to generate geostrophic motion in the ocean. That is to say that when a front becomes stationary or falls below a critical speed, the wind stresses acting on an ocean surface will generate an Ekman layer along with internal gravity waves and surface waves. The Ekman layer generated has characteristic $(v, u)$ velocities of the order

$\left(u_{\mathrm{E}}, v_{\mathrm{E}}\right) \simeq \frac{\tau_{y}(x, t)}{(v f)^{1 / 2}}$,

where $v$ is the viscosity and an associated vertical velocity of

$w_{\mathrm{E}}=\left(\frac{\partial \tau_{y}}{\partial x}\right)\left(\frac{1}{f}\right)$. 

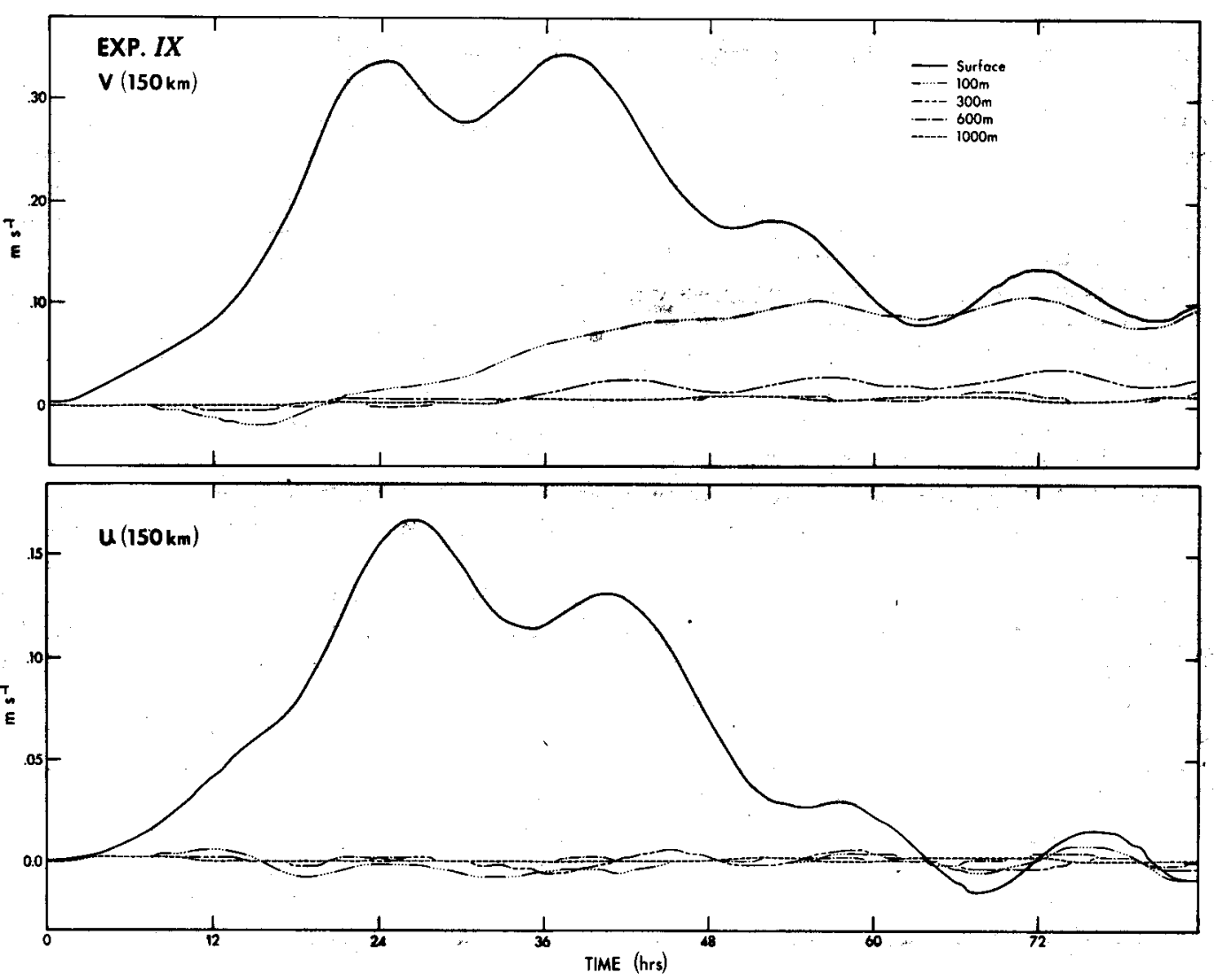

Fig. 6. Time histories of the $v$ and $u$ velocities taken at depths ranging from the surface to $1000 \mathrm{~m}$.

This vertical velocity produces an Ekman pumping that generates considerable vertical motion below the Ekman layer. The circulation, if intense, can generate frontogenesis in the ocean. It is therefore easy to see that for an atmospheric front to be an effective mechanism for generating an oceanic front, the transitory velocity of the atmospheric front must be considerably smaller than the advective horizontal velocities produced by the Ekman pumping. To achieve this, for wind stresses of $0.4-0.5 \mathrm{~N} \mathrm{~m}^{-2}$ and horizontal scales of 50-100 $\mathrm{km}$, the horizontal velocities in the ocean will be of the order of $0.20 \mathrm{~m} \mathrm{~s}^{-1}$. This implies that the atmospheric front must be practically stationary (less than $0.20 \mathrm{~m} \mathrm{~s}^{-1}$ ) over the ocean for about 1 pendulum day to generate an oceanic front; this perhaps is a severe limitation on the effectiveness of mesoscale atmospheric forcing.

\subsection{Constant stratification}

To proceed with our investigation of the effects of realistic forcings and other environmental Tellus 35A (1983), 4 conditions, it was necessary to simplify the model in two ways. One was to eliminate, as mentioned before, the non-linear terms in all of the equations of motion (Appendix), and the other was to use a constant stratification. The solution here differs from that of Section 3 in that forcing due to the moving cold front is more realistic as far as atmospheric forcing is concerned, and instead of using the very unrealistic mixed layer, we consider an eddy viscosity that has a maximum value at the surface layers. Results from Exp. I (see Table A1) show (Figs. 7a and 7b) the details of the density and velocity fields at two different times (100 and $167 \mathrm{~h}$ ); the storm had already dissipated. The solution is characterized by a sharp density gradient in the upper layers (seen in the top portion of Figs. $7 a$ and $7 b$ ), a geostrophically balanced surface jet perpendicular to the plane, and a superimposed field of internal gravity waves that propagate out from the region of forcing. In Exp. II the Brünt-Väisälä frequency was twice as large as that used in Exp. I. This implies that $R N_{\mathrm{II}}=2 R N_{\mathrm{I}}$ 
a
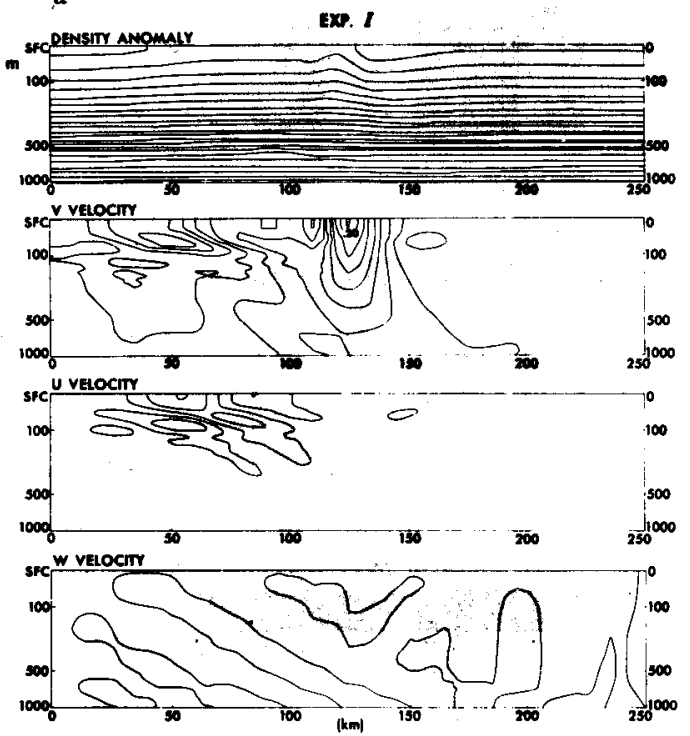

b
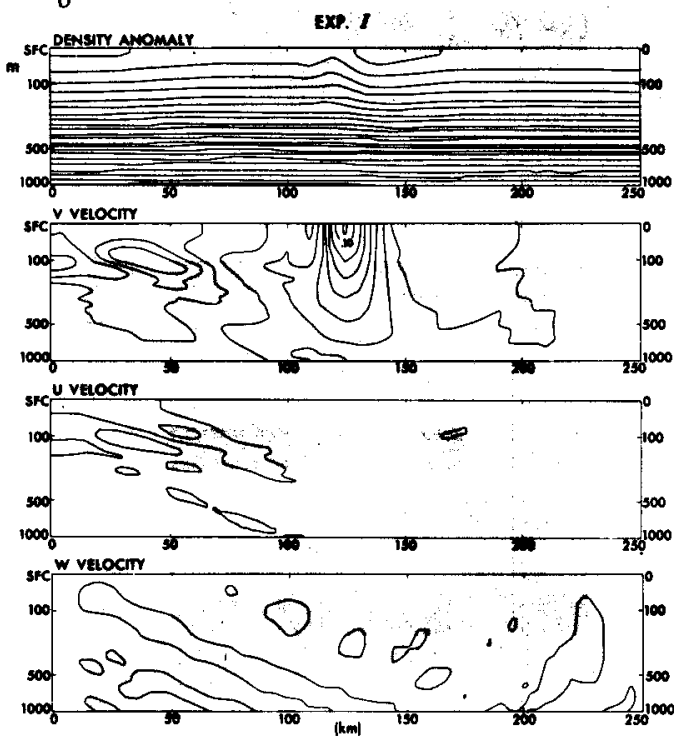

Fig. 7. (a) Contours of the density and velocity fields from Exp. I at $t=100 \mathrm{~h}$. (b) Contours of the density and velocity fields from Exp. I at $t=167 \mathrm{~h}$. Contour intervals in (a) and (b) for $\rho^{\prime}, v, u$, and $w$ are $5 \times 10^{-5}, 2.5 \times 10^{-2}, 2.5$ $\times 10^{-2}$, and $2.5 \times 10^{-4}$, respectively. Maxima for $\rho^{\prime}, v, u$, and $w$ are $7.5 \times 10^{-4} \mathrm{~kg} \mathrm{~m}^{-3}, 0.23 \mathrm{~m} \mathrm{~s}^{-1}, 0.002 \mathrm{~m} \mathrm{~s}^{-1}$, and $0.0003 \mathrm{~m} \mathrm{~s}^{-1}$ at $100 \mathrm{~h}$ and $7.5 \times 10^{-4} \mathrm{~kg} \mathrm{~m}^{-3}, 0.13 \mathrm{~m} \mathrm{~s}^{-1},-0.04 \mathrm{~m} \mathrm{~s}^{-1}$, and $0.00067 \mathrm{~m} \mathrm{~s}^{-1}$ at $167 \mathrm{~h}$.

$=210 \mathrm{~km}$. A comparison of the surface jet profiles can be seen in the lower part of Fig. 8; the graphs in the middle allow us to compare the envelope of the surface heights over a period of $4 \mathrm{~h}$, whereas the upper portion of this figure shows the profile of the wind stresses at their maximum value $(54 \mathrm{~h})$. As we saw before, the horizontal response is the same as the forcing scale regardless of the internal Rossby radius of deformation. If we look at the vertical profiles of the surface jet for these two experiments (Fig. 9), we find that the rate of decay for $V$ in Exp. II is much larger than that in Exp. I, and we can estimate the penetration depth to be around $330 \mathrm{~m}$ for Exp. I and about $157 \mathrm{~m}$ for Exp. II, confirming the fact that the penetration depth in these scales is inversely proportional to $R N$. Also, note that the maximum velocity at the surface in Exp. II is twice as large as that of Exp. I, in agreement with (3.24), since the non-dimensional velocity is equal to $G_{0}$, and in order to dimensionalize it, one must multiply it by $R N f$.

\subsection{Dependence on the forcing scale}

Experiments III, IV, V, and VI use the same internal parameters as those used in Exp. II. One small difference, however, is that these experiments have an exponential stratification in the surface layers. This slight change in the stratification does not seem to have any significant effect on the response, as we shall see later. These experiments exemplify the oceanic response for the different horizontal scales of the forcing. The half-width of the wind stresses are $280 \mathrm{~km}, 210 \mathrm{~km}, 140 \mathrm{~km}$, and $70 \mathrm{~km}$ for Exp. III-VI, respectively. The surface response under these conditions can be seen in Fig. 10. We should point out that the horizontal scale for Exp. III is twice as large as that in Exp. V and four times larger than that of Exp. VI. One prominent feature in these figures is that the surface jets have similar horizontal profiles and also show an increase in amplitude as the scale decreases. The vertical profiles for the four experiments (Fig. 11) are characterized at the maximum response by positive velocities in the first $500 \mathrm{~m}$ and smaller negative velocities below that point. To summarize the results from these four cases, the $v$-velocity amplitude for the permanent response and its half-wideth scale $\left(X_{\mathrm{R}}=\right.$ the distance between the maximum and minimum) are shown in Fig. 12 as a function of the storm scale. It is obvious from this graph that the half-width response scale is linearly related to the half-width storm scale, whereas the maximum velocity is inversely proportional to the storm scale. Notice that the expressions for $V$ 

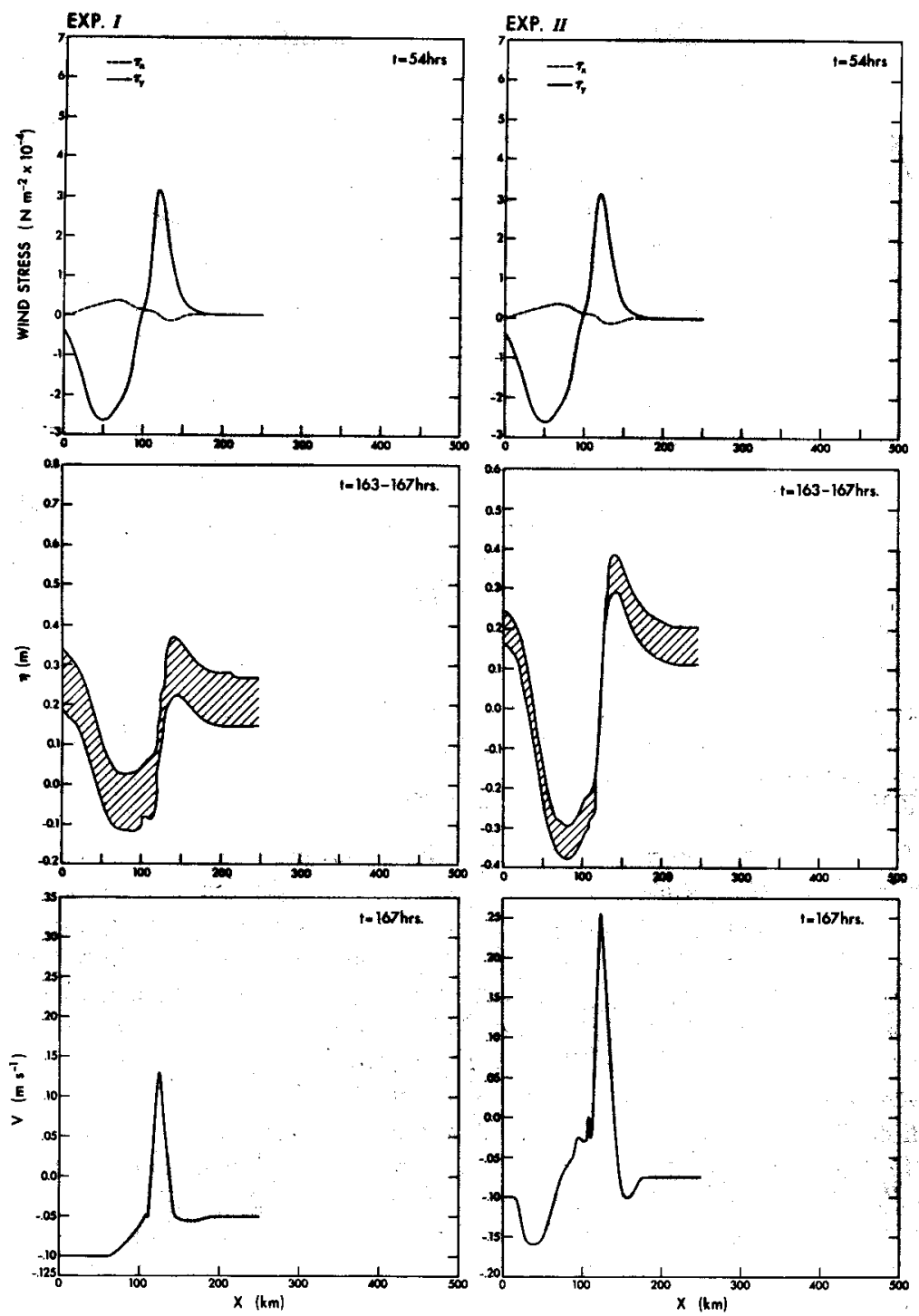

Fig. 8. Results from Exps. I and II showing the maximum wind stresses (upper), the envelope of surface heights over a 4-h period (middle), and the surface jet profiles (lower).

(3.24) and, in fact, for $V_{\mathrm{s}}(3.25)$ are equal to $-G_{0}$. In that example $G_{0}$ remains constant, but we must keep in mind that $G_{0}$ is the non-dimensional amplitude of the curl of the stresses, and we can easily show by integrating (3.11) that for the amplitude to be constant, the stress itself should decrease with $L$. In those numerical experiments, however, in which the stress itself is constant, the amplitude of the velocity should increase inversely proportional to $L$. It is clear from Fig. 11d that the vertical profiles abruptly change at a depth of 100 $\mathrm{m}$, and this is because the upper layers may be controlled by a mixed layer and different higher stratifications (see Table A1 in the Appendix). That being the case, we should calculate the penetration depth for characteristic values above and below the $100 \mathrm{~m}$ level. The characteristic scale $h$ in Fig. 13 refers to layers above $100 \mathrm{~m}$, and $h_{e}$ refers to those layers below $100 \mathrm{~m}$. Since the analytic prediction is calculated from a particular solution (Fig. 4), one 

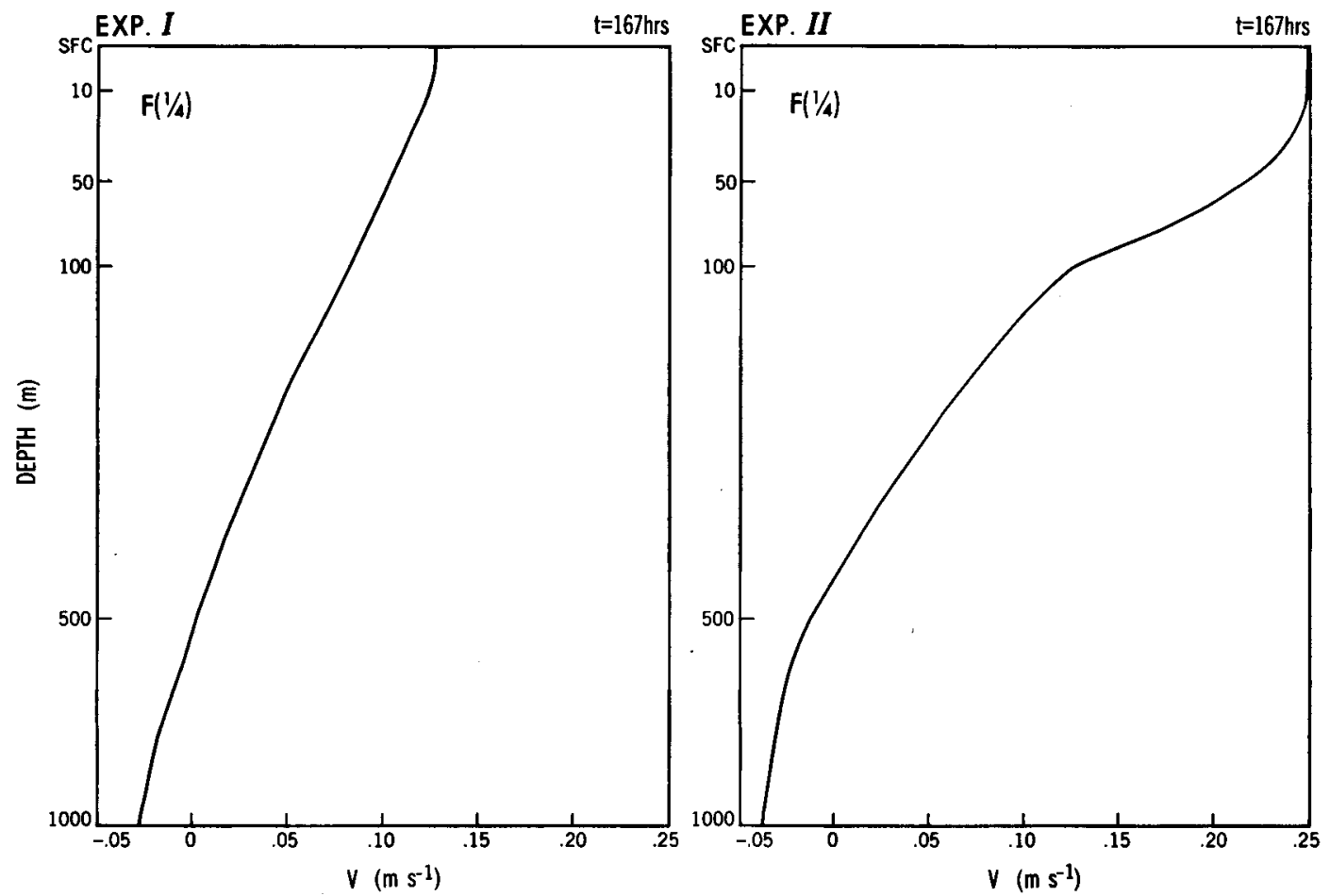

Fig. 9. Vertical profiles of the surface jet for Exps. I and II after $167 \mathrm{~h}$ of integration.
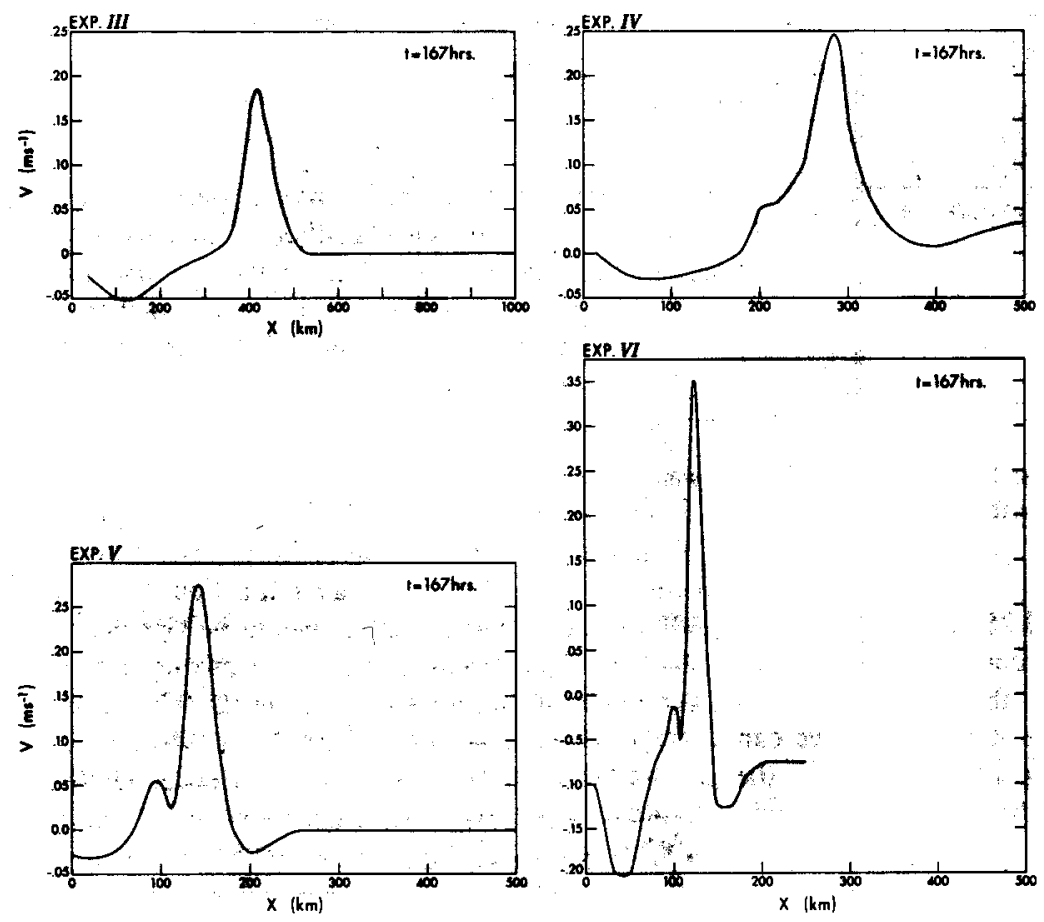

Fig. 10. The surface jet profiles after $167 \mathrm{~h}$ of integration for Exps. III, IV, V, and VI. 


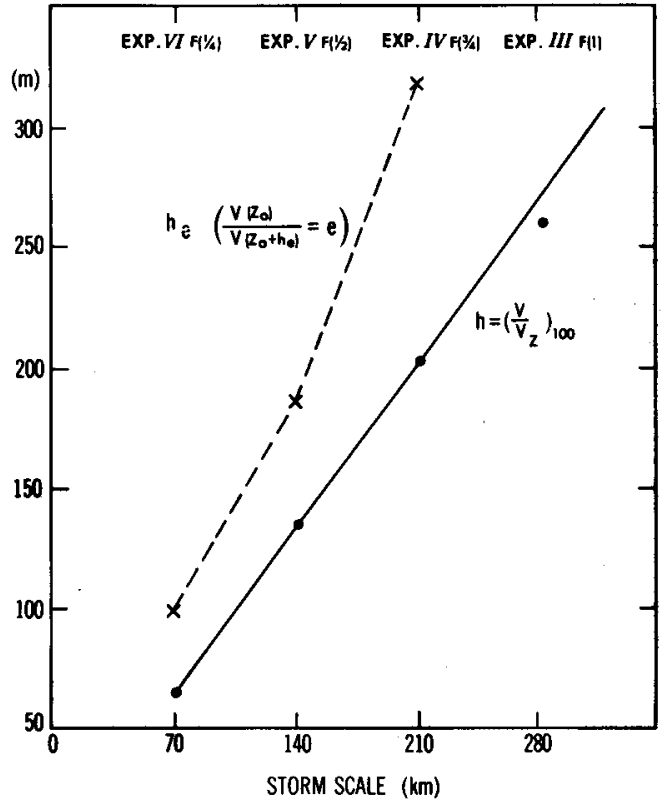

Fig. 13. Penetration depths for Exps. III-VI as a function of the storm scale.

(Exps. VIIa-VIId) had a large viscosity in the boundary layer (boundary layer depths of 27 and $45 \mathrm{~m}$ were used in Exps. VIIb and VIIc, respectively) and a very small interior viscosity, or a constant viscosity (medium and high, Exps. VIIa and VIId respectively) for the entire depth, or a non-linear eddy viscosity as a function of the Richardson number (Exp. VIIc). These solutions, displayed in Fig. 14, show the $v$-velocity contours and eddy-viscosity profiles after $67 \mathrm{~h}$ of integration. The first and most important conclusion one can draw is that the response of the basic features of the geostrophic flow, both in the horizontal and vertical scales, is the same regardless of the turbulent parameterization used. As we might expect, the maximum intensity of the surface jet is inversely proportional to the local value of the viscosity. It is also apparent from the solutions that the intensity of the wavelike disturbances is inversely proportional to the viscosity in the interior layers. Experiment VIIe, on the other hand, shows how the surface response can be affected by an inhomogeneous eddy viscosity. Since the viscosity is a function of the Richardson number (Fig. 14e shows only a typical profile), one might expect that the mixed layer below the maximum wind stress would be deeper than in its surroundings, thus producing by itself a shallow response superimposed on the direct mechanical response. The numerical solution, however, shows this effect to be only a few percent of the total response.

\section{Non-linear response}

A review of studies of non-linear effects in the geostrophic adjustment problem can be found in Blumen (1972), in which the possibility that non-linear solutions may exhibit or develop hydraulic jumps was discussed. Those studies (Tepper, 1955) had an adjustment to particular initial conditions, whereas in our case this possibility is not found because of the transient forcing. Let us then review the non-linear terms which are present in the study. Basically, all of the advection terms in the vorticity, momentum, and density equations are non-linear (see equations Al-A4). Some cases include non-linear terms in which the eddy viscosity and diffusivity are dependent on the local Richardson number. This effect has been already discussed in some of the linear experiments of the previous section (Fig. 14); it is therefore not regarded as a new non-linear term in the present solutions under consideration. Essentially then, the difference between the solutions presented here and those of the previous section lies in the advection terms in the equations of motion.

\subsection{Constant stratification}

For simplicity, the effect of the non-linear terms will be presented in the same setting as that used for Exp. I. Density and velocity fields at two different times (100 and $167 \mathrm{~h}$ ) are shown in Fig. 15a and $15 \mathrm{~b}$, respectively, for the non-linear Experiment VIII, and should be compared with the linearized solution of Exp. I (Figs. 7a and 7b). At first glance the solutions look quite similar. The density pattern in both cases shows a similar surface front; the $v$ velocity seems different, however, in that the linear one seems to have sharper gradients. The $v$ velocity is actually stronger in Exp. VIII, and the presence of waves is more noticeable (Figs. 7a and 15a), especially in the $u$-velocity field. At later times the similarities persist and the waviness is also present. Profiles of the wind stresses, the surface heights, and the surface $v$ velocity are shown in Fig. 16. Upon comparing this figure with the profiles shown on the left side of Fig. 8, we find that the main difference is in the intensity of the jet rather than its 

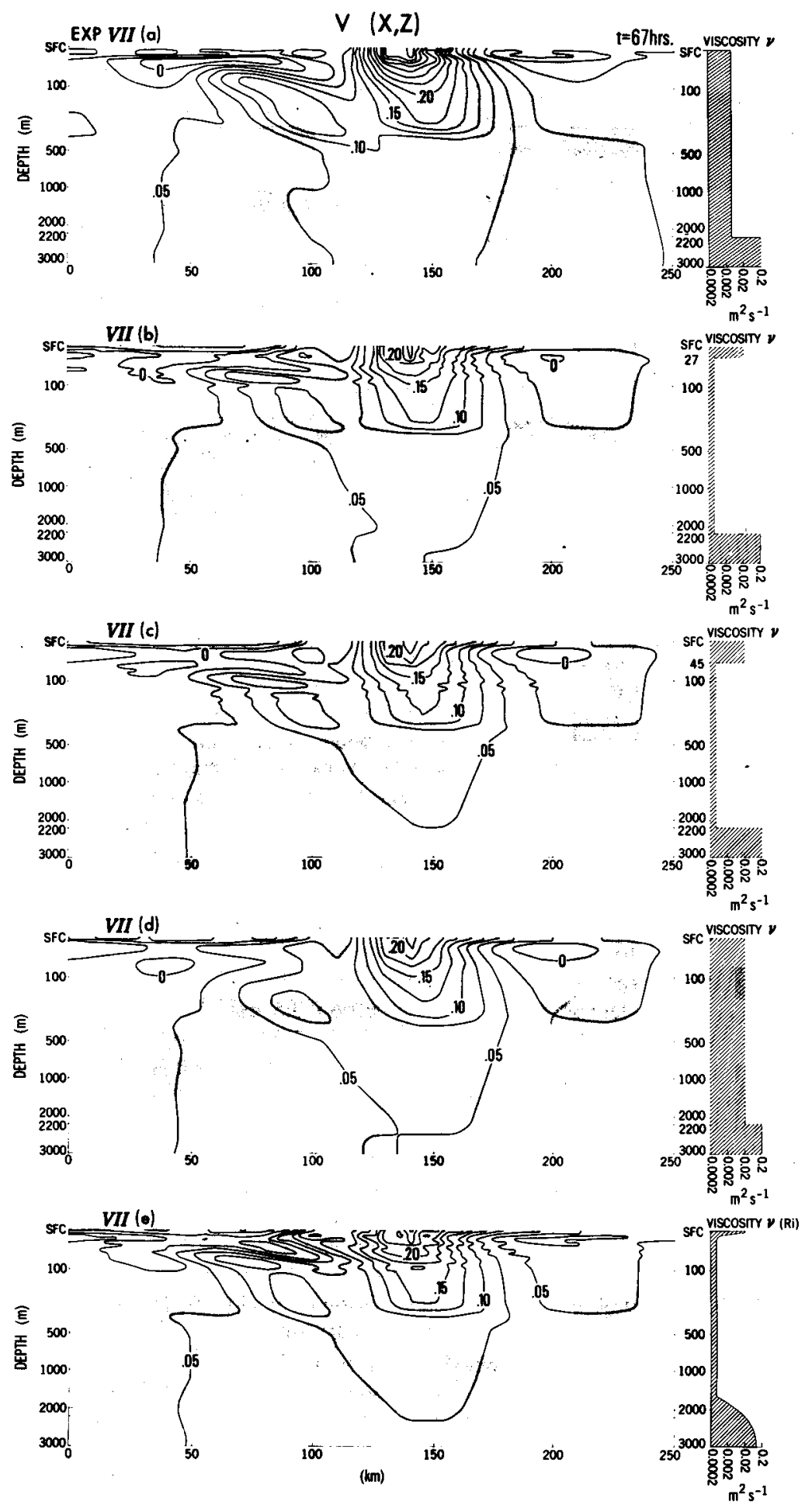

Fig. 14. Contours of the $v$ velocity after $67 \mathrm{~h}$ of integration and the eddy viscosity vertical profile for Exps. VIIa-VIIe. Maximum velocities are $0.42,0.26,0.24,0.27 \mathrm{~m} \mathrm{~s}^{-1}$ respectively.

Tellus 35A (1983), 4 
a
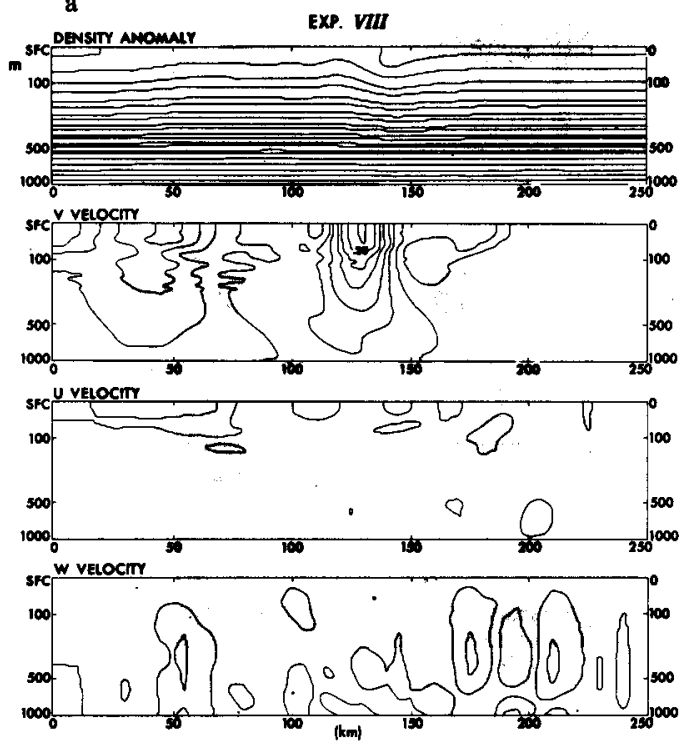

b
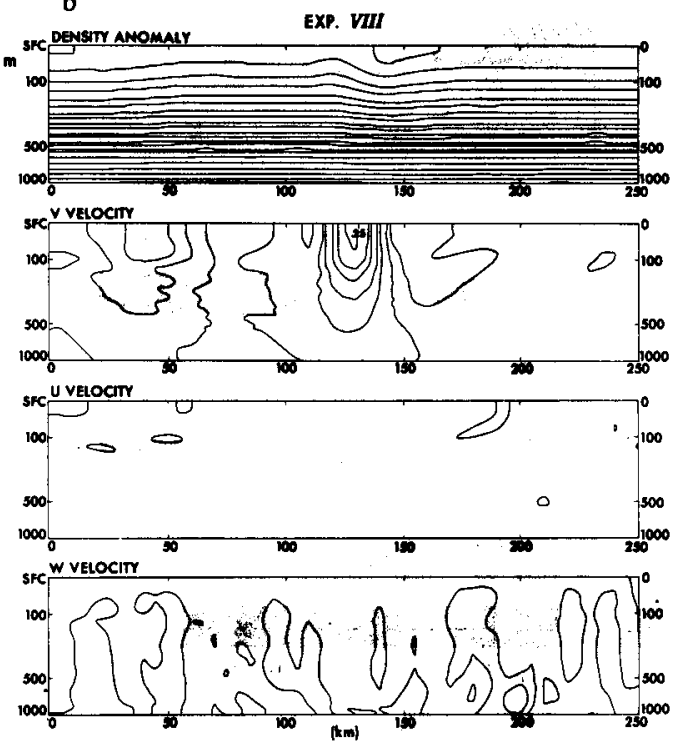

Fig. 15. (a) Contours of the density and velocity fields from Exp. VIII at $t=100 \mathrm{~h}$. (b) Contours of the density and velocity fields from Exp. VIII at $t=167 \mathrm{~h}$. Contour intervals in (a) and (b) for $\rho^{\prime}, v, u$, and $w$ are $5 \times 10^{-5}, 2.5 \times 10^{-2}$, $2.5 \times 10^{-2}$ and $2.5 \times 10^{-4}$, respectively. Maxima for $\rho^{\prime}, v, u$, and $w$ are $7.5 \times 10^{-4} \mathrm{~kg} \mathrm{~m}^{-3}, 0.23 \mathrm{~m} \mathrm{~s}^{-1}, 0.008 \mathrm{~m} \mathrm{~s}^{-1}$, and $0.00047 \mathrm{~m} \mathrm{~s}^{-1}$ at $100 \mathrm{~h}$ and $7.5 \times 10^{-4} \mathrm{~kg} \mathrm{~m}^{-3}, 0.26 \mathrm{~m} \mathrm{~s}^{-1},-0.046 \mathrm{~m} \mathrm{~s}^{-1}$, and $0.00042 \mathrm{~m} \mathrm{~s}^{-1}$ at $167 \mathrm{~h}$.

width, i.e., it ranges from $0.25 \mathrm{~m} \mathrm{~s}^{-1}$ to $0.04 \mathrm{~m} \mathrm{~s}^{-1}$ for Exp. VIII and $0.14 \mathrm{~m} \mathrm{~s}^{-1}$ to $0.1 \mathrm{~m} \mathrm{~s}^{-1}$ for Exp. I. The difference certainly becomes more noticeable when we compare the vertical profiles of the $v$ velocity of Exps. VIII and I (Figs. 17 and 9 respectively). The major difference here is that the penetration depth for the non-linear solution seems greater than in the linear case. Also related to this difference is that the mean velocity seems larger in the non-linear case, and that the advection terms somehow inhibit the generation of waves that prevail in the linear solution, thus making the geostrophic adjustment in the non-linear case more effective. It is as though the finely tuned resonant conditions by which waves grow in the linear regime are altered by horizontal advection in the boundary layer, reducing the wave energy available to interfere with the adjustment.

\subsection{Variable stratification}

The diversity of density-profile characteristics in the world ocean, as well as the seasonal variability in the upper layers of the oceans, makes it difficult to extrapolate the response of cases with constant stratifications to those with realistic conditions. Two drastically different extreme conditions were used for Exps. IX and X: one with a steep shallow thermocline characteristic of a summer thermocline (Exp. IX) and the other a smooth deep thermocline (Exp. X). In both cases the viscosity was constant $\left(v=0.013 \mathrm{~m}^{2} \mathrm{~s}^{-1}\right)$. The solutions after $84 \mathrm{~h}$ of integration are shown in Fig. 18. Briefly summarizing the differences between these experiments, we can see that the shallow thermocline produces a slightly more intense and shallow jet than that of Exp. X. These results are in agreement with our discussion in Section 3.5 concerning penetration depth as a function of stratification if one takes account of the average stratification that prevails in the upper layers of the ocean.

\section{Summary}

The present study shows that atmospheric forcing can be an important source of mesoscale variability in the ocean. The time scale required for an atmospheric storm to be effective in generating a permanent, geostrophically balanced response in the ocean is in the order of 1 day. Moreover, we have shown that the response is linearly proportional to the product of the time scale of the storm and its intensity.

Atmospheric fronts are strong candidates for mesoscale forcing, but they are certainly not the 

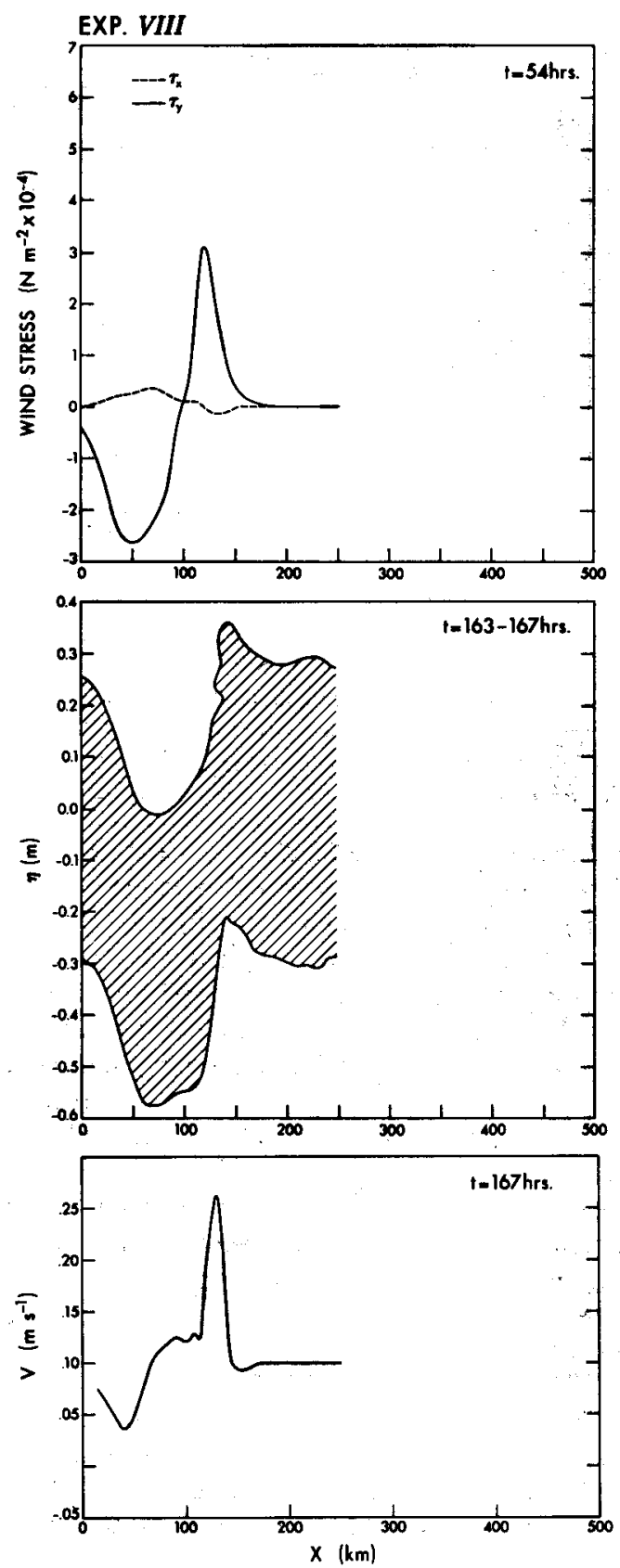

Fig. 16. Profiles of the wind stresses, surface heights, and the surface jet for Exp. VIII.

only ones. Other phenomena that might also be important sources of forcing include squall lines with large surface vorticities, cloud clusters, and meso-convective complexes. One might conceive

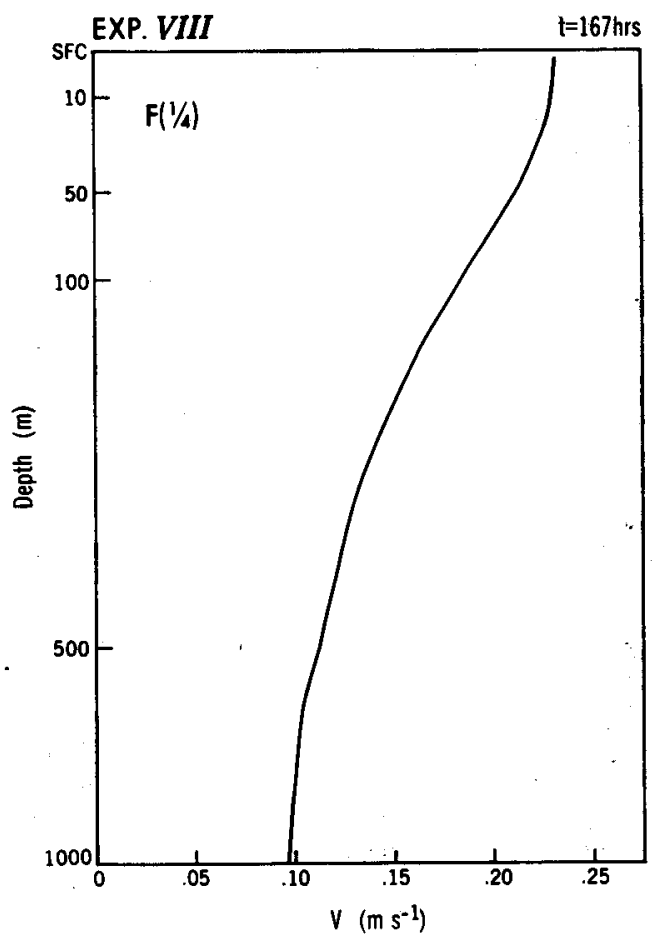

Fig. 17. Vertical profile of the $v$ velocity for Exp. VIII at $t=167 \mathrm{~h}$. Note that the forcing for Exp. VII was $F=$ $F / 4$.

that even an intense thunderstorm could produce a significant signature in the ocean if it existed long enough. This is somewhat unlikely, however, because although such small-scale phenomena may be intense, their lifetimes are no more than a few hours.

The role of the Rossby radius of deformation in determining the oceanic response has been clarified by this study. We emphasize that for storms with scales considerably smaller than the barotropic Rossby radius of deformation, the oceanic stratification and the horizontal extent of the storm are the only factors that determine the penetration depth of the response, which means that it is not the Rossby radius of deformation but rather the scale of penetration depth $(h=(f / N) L)$ that characterizes the response.

In exploring the effect of differing eddy-viscosity parameterization, we did not find any significant differences in the qualitative comparison of the results, although, as one might expect, we did find quantitative differences. 
EXP. IX

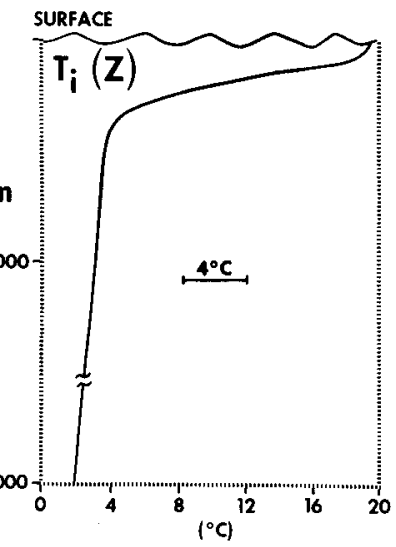

EXP. $X$

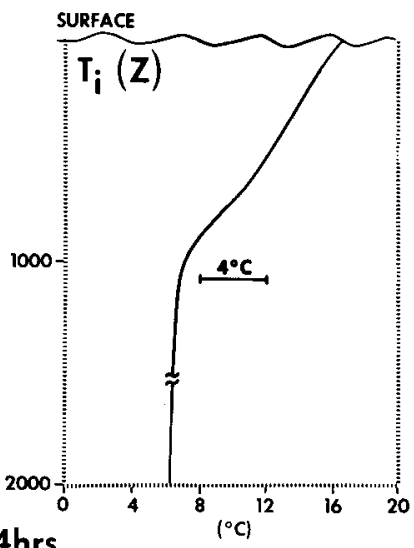

$t=84 \mathrm{hrs}$
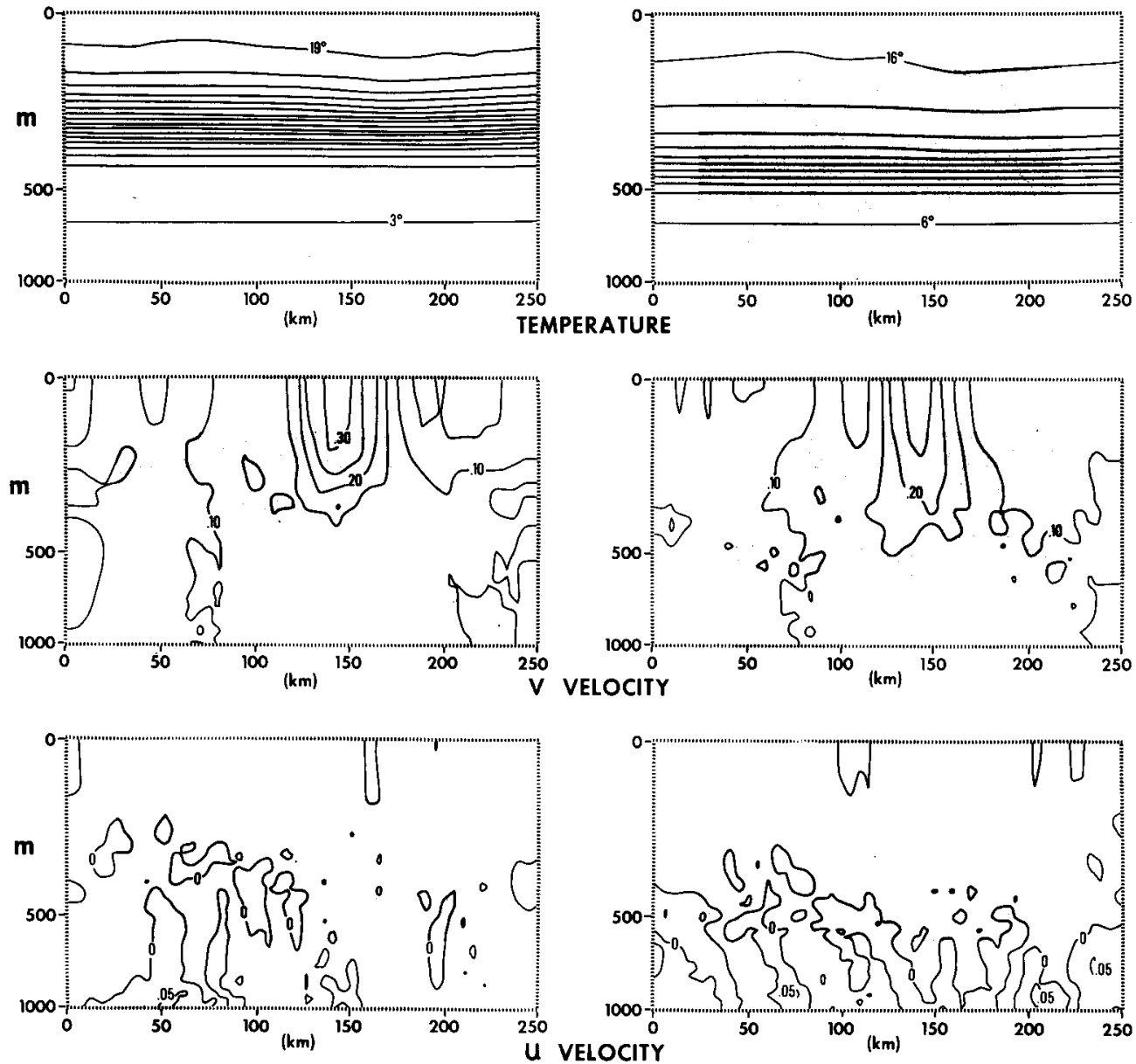

Fig. 18. Results from Exp. IX and $\mathrm{X}$ at $t=84 \mathrm{~h}$ showing the thermocline profile and contours of the temperature, $v$ velocity and $u$ velocity. 
The role of the mixed layer is considered very important in the transfer of surface stresses down into the system. The mixed layer does not seem to be important, however, in determining the characteristic length of the problem, that is, at least for storms that give a penetration depth considerably larger than the mixed layer (for a mixed layer on the order of $20 \mathrm{~m}$, the storm should be larger than a few kilometers).

The non-linear advection terms seem to affect the geostrophic adjustment process more by reducing the associated wave energy than by modifying the characteristics of the geostrophic response.

Finally, we have also demonstrated that making the stratification more realistic does not significantly alter the results.

It is easy to foresee that the suggestions in this paper concerning the effectiveness of atmospheric forcing can be verified with present technology. For that matter, studies may already exist that support this suggestion. One approach might be to monitor ocean temperatures inferred by satellites in order to determine whether atmospheric fronts can generate thermal gradients in the ocean. Unfortunately, the present temperature resolution is inadequate for detecting the response in most cases, but some extreme events may be observed.

\section{Acknowledgements}

Some of the material of this paper was intended to be part of the Centennial Issue of the Meterological Society of Japan. Unfortunately, we were unable to meet that deadline. We wish, however, to extend our congratulations to the members of this distinguished society.

The impetus to complete this study resulted from the encouragement and lengthy discussions with Dr. George Philander to whom the senior author is especially thankful. We would also like to thank Dr. Bruce Ross for his assistance in setting up the interface between the atmospheric and oceanic models. In addition, we extend our appreciation to Mr. R. Shaginaw for his editorial assistance, to Mr. $\mathrm{K}$. Moore for verifying some of the mathematical equations, to Messrs. P. Tunison, W. Ellis, M. Zadworney and J. Conner for preparing the figures, and to Mrs. Betty Williams for typing the manuscript.

\section{Appendix. The numerical model}

The model used in this study is merely an extension of the model described in Orlanski and Ross (1973), Orlanski et al. (1974), Orlanski (1976), and Orlanski and Ross (1977). We refer the reader to those papers for specific details and we shall discuss only the changes needed to accomplish the present study.

The primary difference is that we have changed from an atmospheric model to an oceanic model which as in the former case employs either the "deep anelastic" equations as formulated by Ogura and Phillips (1962) or a hydrostatic approximation of these equations. It should be noted that references to $\theta$ variables in the equations are density variables in this case. The equations are written in Cartesian coordinates $(x, y)$, but for simplicity we assume that the predicted quantities do not depend on the $y$ coordinate. We retain the $v$ velocity for rotational effects. Then using the set of equations as defined in Orlanski and Ross (1977) our non-linear set of equations are:

$$
\begin{aligned}
& \frac{\partial \zeta}{\partial t}-J\left(\psi, \alpha_{0} \zeta\right)=f \frac{\partial v}{\partial z}-\frac{g}{\theta_{0}} \frac{\partial \theta}{\partial x}+\frac{\partial \theta}{\partial x}\left(K v_{\mathrm{e}} \frac{\partial \zeta}{\partial x}\right) \\
& +\frac{\partial}{\partial z}\left(v_{\mathrm{e}} \frac{\partial \zeta}{\partial z}\right) \\
& \frac{\partial v}{\partial t}-\alpha_{0} J(\psi, v)=-f\left(\alpha_{0} \frac{\partial \psi}{\partial z}-U_{\mathrm{g}}\right) \\
& \quad+\frac{\partial}{\partial x}\left(K v_{\mathrm{e}} \frac{\partial v}{\partial x}\right)+\frac{\partial}{\partial z}\left(v_{\mathrm{e}} \frac{\partial v}{\partial z}\right) \\
& \frac{\partial \theta}{\partial t}-\alpha_{0} J(\psi, \theta)+V \frac{\partial \theta_{g}}{\partial y}=\frac{\partial}{\partial x}\left(K \kappa_{\mathrm{e}} \frac{\partial \theta}{\partial x}\right) \\
& \quad+\frac{\partial}{\partial z}\left(\kappa_{\mathrm{e}} \frac{\partial \theta}{\partial z}\right)
\end{aligned}
$$

and

$\zeta=a_{0} \frac{\partial^{2} \psi}{\partial x^{2}}+\frac{\partial}{\partial z}\left(\alpha_{0} \frac{\partial \psi}{\partial z}\right)$

where the Jacobian is defined as

$J(A, B)=(\partial A / \partial x) \frac{\partial B}{\partial z}-(\partial A / \partial z) \frac{\partial B}{\partial z}$ 
If we then neglect the Jacobian terms, our linearized equations become

$$
\begin{aligned}
\frac{\partial \zeta}{\partial t} & =f \frac{\partial v}{\partial z}-\frac{g}{\theta} \frac{\partial \theta}{\partial x}+\frac{\partial}{\partial x}\left(K v_{\mathrm{e}} \frac{\partial \zeta}{\partial x}\right) \\
& +\frac{\partial}{\partial z}\left(v_{\mathrm{e}} \frac{\partial \zeta}{\partial z}\right), \\
\frac{\partial v}{\partial t} & =-f\left(a_{0} \frac{\partial \psi}{\partial z}-U_{\mathrm{s}}\right)+\frac{\partial}{\partial x}\left(K v_{\mathrm{e}} \frac{\partial v}{\partial x}\right) \\
& +\frac{\partial}{\partial z}\left(v_{\mathrm{e}} \frac{\partial v}{\partial z}\right), \\
\frac{\partial \theta}{\partial t}+a_{0}\left(\frac{\partial \psi}{\partial x} \frac{\partial \bar{\theta}}{\partial z}\right)+V \frac{\partial \theta_{\mathrm{g}}}{\partial y}=\frac{\partial}{\partial x}\left(K \kappa_{\mathrm{e}} \frac{\partial \theta}{\partial x}\right) & \\
& +\frac{\partial}{\partial z}\left(\kappa_{\mathrm{e}} \frac{\partial \theta}{\partial z}\right),
\end{aligned}
$$

and

$\zeta=a_{0} \frac{\partial^{2} \psi}{\partial x^{2}}+\frac{\partial}{\partial z}\left(\alpha_{0} \frac{\partial \psi}{\partial z}\right)$,

where $\alpha_{0}$ is the specific volume and is defined as $\alpha_{0}$ $=1 / \rho_{0}$. Studies have shown that the hydrostatic assumption is a good approximation where the horizontal velocities are much larger than vertical velocities. This assumption is also computationally more economical than the ADI (alternating direction implicit) method of solving the Poisson equation; therefore, if we utilize these facts, eq. (A4) becomes

$\zeta=\frac{\partial}{\partial z}\left(a_{0} \frac{\partial \psi}{\partial z}\right)$.

Rather than using the method outlined in Orlanski and Ross (1977) for computing the eddy diffusivity, $\kappa_{\mathrm{e}}$, we use a method where $\kappa_{\mathrm{e}}$ is a function of the Richardson number

$\kappa_{\mathrm{e}}=\kappa_{0} 10^{(0.9(1-\tanh (R l-1))}$ for $J>13$,

and

$\kappa_{\mathrm{e}}=\kappa_{\mathrm{e}}\left(1+100 \mathrm{e}^{\left((J-3)^{2} / 25\right)}\right) \quad$ for $J \leq 13$,

where the Richardson number is

$R i=\frac{g(\partial \theta / \partial z)}{(\partial u / \partial z)^{2}+(\partial v / \partial z)^{2}}$, and is limited to $-10 \geq R i \leq 10$ for computational purposes. $J$ is a grid point in the $z$ direction. In some of the later cases we simplified the method by defining constant values for $\kappa_{\mathrm{e}}$ for specific layers. A typical example is

$\kappa_{\mathrm{e}}=0.5 \times 10^{2} \kappa_{0} ; \quad J<8$,

$\kappa_{\mathrm{e}}=\kappa_{0} ; \quad 8 \geq J \leq 58$,

and

$\kappa_{\mathrm{e}}=0.4 \times 10^{1} \kappa_{0} ; \quad J>58$.

Representative values of $K$ and $\kappa_{0}$ are 1.0 and $5 \mathrm{~m}^{2}$ $\mathrm{s}^{-1}$.

\subsection{Domain size and model resolution}

The domain has a grid system with 51 uniformly spaced points in the $x$ direction and 61 points with variable spacing in the $z$ direction. Representative values for $\Delta x$ are 5,10 , and $20 \mathrm{~km}$, depending on the particular case, respectively, implying domain lengths of 250,500 and $1000 \mathrm{~km}$. The depth remained fixed for all the cases at $3000 \mathrm{~m}$ and had a $\Delta z$ of about $7 \mathrm{~m}$ near the surface and a $\Delta z \approx 133$ $\mathrm{m}$ at the bottom. The atmospheric boundary is $1500 \mathrm{~km}$ in length, equally spaced, with a $\Delta x_{\mathbf{F}}=20$ $\mathrm{km}$ over its 76 points in the $x$ direction. The atmospheric disturbance has no grid points in the $y$ or $z$ direction. The $\Delta x_{F}$ also varied with respect to the $\Delta x$ used for the ocean domain, i.e., when $\Delta x=$ $10 \mathrm{~km}$, then $\Delta x_{\mathrm{F}}=10 \mathrm{~km}$. The solution was time-smoothed to suppress mode splitting associated with the leapfrog method used.

\subsection{Boundary conditions}

During the life of the frontal disturbance we utilize the atmospheric values of pressure, $u$, and $v$ for boundary conditions at the air-sea interface. The $x$ velocity is

$u_{t}=f v-\alpha p_{x}+\frac{\partial t^{(x)}}{\partial z}$

with $p$ defined as

$p=p_{\mathrm{a}} g \rho_{\mathrm{B}} \eta+g\left(\rho_{\mathrm{B}}(1-\theta)\right) \frac{\Delta z}{2}$,

so that

$\alpha p_{x}=\alpha p_{\mathrm{a}_{x}}+\alpha g \rho_{\mathrm{B}} \eta_{x}-\alpha g \frac{\Delta z}{2} \rho_{\mathrm{B}} \theta_{x}$ 
Table A1

\begin{tabular}{|c|c|c|c|c|c|}
\hline Experiment & $\begin{array}{l}L(\mathrm{~km}) \\
\text { (forcing scale) }\end{array}$ & $(R N(\mathrm{~km})$ & Linear & Eddy viscosity $\left(\mathrm{m}^{2} \mathrm{~s}^{-1}\right)$ & Stratification \\
\hline I & 70 & 105 & Yes & $\begin{array}{l}0.02 ; Z=S F C-27 \mathrm{~m} \\
0.0005 ; 28 \mathrm{~m}-2315 \mathrm{~m} \\
0.25 ; 2316 \mathrm{~m}-3000 \mathrm{~m}\end{array}$ & Constant $(\gamma=0.00000125)$ \\
\hline II & 70 & 210 & Yes & $\begin{array}{l}0.02 ; Z=S F C-27 \mathrm{~m} \\
0.0005 ; 28 \mathrm{~m}-2315 \mathrm{~m} \\
0.25 ; 2316 \mathrm{~m}-3000 \mathrm{~m}\end{array}$ & Constant $(\gamma=0.000005)$ \\
\hline III & 280 & 210 & Yes & $\begin{array}{l}0.02 ; Z=S F C-27 \mathrm{~m} \\
0.0005 ; 28 \mathrm{~m}-2315 \mathrm{~m} \\
0.25 ; 2316 \mathrm{~m}-3000 \mathrm{~m}\end{array}$ & $\begin{array}{l}\text { Constant }(\gamma=0.000005) \\
\text { exponential stratification in the } \\
\text { upper layers }\end{array}$ \\
\hline IV & 210 & 210 & Yes & $\begin{array}{l}0.02 ; Z=S F C-27 \mathrm{~m} \\
0.0005 ; 28 \mathrm{~m}-2315 \mathrm{~m} \\
0.25 ; 2316 \mathrm{~m}-3000 \mathrm{~m}\end{array}$ & $\begin{array}{l}\text { Constant }(\gamma=0.000005) \\
\text { exponential stratification in the } \\
\text { upper layers }\end{array}$ \\
\hline V & 140 & 210 & Yes & $\begin{array}{l}0.02 ; Z=S F C-27 \mathrm{~m} \\
0.0005 ; 28 \mathrm{~m}-2315 \mathrm{~m} \\
0.25 ; 2316 \mathrm{~m}-3000 \mathrm{~m}\end{array}$ & $\begin{array}{l}\text { Constant }(\gamma=0.000005) \\
\text { exponential stratification in the } \\
\text { upper layers }\end{array}$ \\
\hline VI & 70 & 210 & Yes & $\begin{array}{l}0.02 ; Z=S F C-45 \mathrm{~m} \\
0.0005 ; 28 \mathrm{~m}-2315 \mathrm{~m} \\
0.25 ; 2316 \mathrm{~m}-3000 \mathrm{~m}\end{array}$ & $\begin{array}{l}\text { Constant }(\gamma=0.000005) \\
\text { exponential stratification in the } \\
\text { upper layers }\end{array}$ \\
\hline VIIa & 140 & 51.5 & Yes & $\begin{array}{l}\text { Function of a constant } \\
R i \#=1.0\end{array}$ & $\begin{array}{l}\text { Constant }(\gamma=0.0000003) \\
\text { exponential stratification in the } \\
\text { upper layers }\end{array}$ \\
\hline VIIb & 140 & 51.5 & Yes & $\begin{array}{l}0.02 ; Z=S F C-27 \mathrm{~m} \\
0.0005 ; 28 \mathrm{~m}-2315 \mathrm{~m} \\
0.25 ; 2316 \mathrm{~m}-3000 \mathrm{~m}\end{array}$ & $\begin{array}{l}\text { Constant }(\gamma=0.0000003) \\
\text { exponential stratification in the } \\
\text { upper layers }\end{array}$ \\
\hline VIIc & 140 & 51.5 & Yes & $\begin{array}{l}0.02 ; Z=S F C-27 \mathrm{~m} \\
0.0005 ; 46 \mathrm{~m}-2315 \mathrm{~m} \\
0.25 ; 2316 \mathrm{~m}-3000 \mathrm{~m}\end{array}$ & $\begin{array}{l}\text { Constant }(\gamma=0.0000003) \\
\text { exponential stratification in the } \\
\text { upper layers }\end{array}$ \\
\hline VIId & 140 & 51.5 & Yes & $\begin{array}{l}\text { Function of a constant } \\
R i \#=0.0\end{array}$ & $\begin{array}{l}\text { Constant }(\gamma=0.0000003) \\
\text { exponential stratification in the } \\
\text { upper layers }\end{array}$ \\
\hline VIIe & 140 & 51.5 & Yes & $\begin{array}{l}\text { Function of a variable } \\
R i \#\end{array}$ & $\begin{array}{l}\text { Constant }(\gamma=0.0000003) \\
\text { exponential stratification in the } \\
\text { upper layers }\end{array}$ \\
\hline VIII & 70 & 105 & No & $\begin{array}{l}0.02 ; Z=S F C-27 \mathrm{~m} \\
0.0005 ; 28 \mathrm{~m}-2315 \mathrm{~m} \\
0.25 ; 2316 \mathrm{~m}-3000 \mathrm{~m}\end{array}$ & Constant $(\gamma=0.00000125)$ \\
\hline IX & 140 & 51.5 & No & $\begin{array}{l}\text { Function of a constant } \\
R i \#\end{array}$ & $\begin{array}{l}\text { Constant }(\gamma=0.0000003) \\
\text { exponential stratification in the } \\
\text { upper layers }\end{array}$ \\
\hline $\mathbf{X}$ & 140 & 51.5 & No & $\begin{array}{l}\text { Function of a constant } \\
R i \text { \# }\end{array}$ & $\begin{array}{l}\text { Constant }(\gamma=0.0000003) \\
\text { exponential stratification in the } \\
\text { upper layers }\end{array}$ \\
\hline
\end{tabular}

which reduces to

$\alpha p_{x}=\alpha p_{\mathrm{a}_{x}}+g\left(\eta_{x}-\frac{\Delta z}{2} \theta_{x}\right)$,

by the ocean. For the stress terms we have

$\frac{\partial \tau^{(x)}}{\partial z}=\frac{\tau_{\mathrm{SFC}}^{(x)}-\tau_{z=}^{(x)}-\Delta z}{\Delta z}$

$\frac{\partial \tau^{(x)}}{\partial z}=\frac{1}{\Delta z}\left(\tau_{\mathrm{SFC}_{1}}^{(x)}-\left.v_{\mathrm{e}} \frac{\partial u}{\partial z}\right|_{z=-\Delta z}\right)$

where $p_{x}$ represents the hydrostatic pressure along the $x$ direction incurred from the atmosphere, and the second term is the hydrostatic pressure exerted 
Since $\zeta_{1, \mathrm{~J}}=\partial u / \partial z-\partial w / \partial x$, and since the hydrostatic assumption reduces the vorticity term to

$\zeta_{\mathrm{I}, \mathrm{J}}=\frac{\partial u}{\partial z}$

then

$$
\begin{aligned}
u_{t} & =f v-\alpha p_{\mathrm{ax}}-g\left(\eta_{x}-\frac{\Delta z}{2} \theta_{x}\right) \\
& +\frac{1}{\Delta z_{\mathrm{SFC}}}\left(\tau_{\mathrm{SFC}}^{x}-v_{\mathrm{e}} \zeta_{\mathrm{I}, \mathrm{JM}-1}\right) .
\end{aligned}
$$

We then use $u_{1, \mathrm{JM}}$ for the boundary condition on the stream function

$\psi_{\mathrm{I}, \mathrm{JM}}-\psi_{\mathrm{I}, \mathrm{JM}-1}=\frac{1}{\Delta z_{\mathrm{SFC}}} u_{\mathrm{I}, \mathrm{JM}}$,

where the JM represents the surface grid point and JM-1 is the next grid point level below the surface. For the $y$ velocity we define the shear stress in $y$ as

$\tau_{\mathrm{SFC}}^{(y)}=\kappa_{\mathrm{e}} \frac{\partial v}{\partial z}$.

Then at the top boundary we have

$$
V_{\mathrm{I}, \mathrm{JM}}-V_{\mathrm{I}, \mathrm{JM}-1}=\frac{1}{\Delta z_{\mathrm{SFC}}} \tau_{\mathrm{SFC}_{\mathrm{I}}}^{(y)} / \kappa_{\mathrm{e}} .
$$

The stresses are calculated using the atmospheric $u$ and $v$ velocities and are defined in the following manner.

$C_{\mathrm{D}}=\frac{\tau}{\rho_{\mathrm{a}}\left(\left(u_{\mathrm{a}}^{2}+v_{\mathrm{a}}^{2}\right)^{1 / 2}\right)^{2}}$,

so that

$\tau^{(x)}=C_{\mathrm{D}} \rho_{\mathrm{a}} V u_{\mathrm{a}}$,

and

$\tau^{(y)}=C_{\mathrm{D}} \rho_{\mathrm{a}} V v_{\mathrm{a}}$

where $\rho_{\mathrm{a}}$ is the density of the air, $u_{\mathrm{a}}$ and $v_{\mathrm{a}}$ are the atmospheric $u$ and $v$ velocity components and $V=$ $\left(u_{\mathrm{a}}^{2}+v_{\mathrm{a}}^{2}\right)^{1 / 2}$. Values used for the reference densities, namely $\rho_{\mathrm{a}}$ (atmospheric) and $\rho_{\mathrm{B}}$ (ocean boundary) are $1.17 \mathrm{~kg} \mathrm{~m}^{-3}$ and $1000 \mathrm{~kg} \mathrm{~m}^{-3}$ respectively. Drag coefficient values were obtained from Heller- man (1967) and are defined in the following manner:

$C_{\mathrm{D}}=0.0008$ for $V \leq 6.6 \mathrm{~m} \mathrm{~s}^{-1}$

and

$C_{\mathrm{D}}=0.0026$ for $V>6.6 \mathrm{~m} \mathrm{~s}^{-1}$.

As the front passes through the domain, the atmospheric variables undergo a frontolytic process in which we control their intensity by using a time fade function, $T_{\mathrm{F}}$,

$$
\begin{aligned}
& T_{\mathrm{F}}=\left(\tanh \left(\left(T+T_{0}\right) / 10^{4}\right)\right. \\
& \quad \times\left(0.5\left(1-\tanh \left(\left(T+10^{4}\right) / 8 \times 10^{4}\right)\right) .\right.
\end{aligned}
$$

where $T_{0}(50 \mathrm{~h})$ is the initial time for the frontal position with respect to the oceanic grid and the elapsed time, $T$ is

$T=m \Delta t-T_{0}$,

with $m$ being the number of time steps and $\Delta t$ the model time step (30 s). To calculate the frontal position, $X_{\mathrm{F}}$, we specify the front to be contained in the domain between 20 and $30 \mathrm{~h}$ of integration at which time the variables are at their maximum intensity. Therefore, we define a beginning and stopping time and calculate the location relative to those times. They are

$T_{\mathrm{B}}=T_{1}-T_{0}=-30 \mathrm{~h}$.

and

$T_{\mathrm{s}}=T_{2}-T_{0}=-20 \mathrm{~h}$,

where $T_{1}$ and $T_{2}$ equal 20 and $30 \mathrm{~h}$ respectively. Then when $T<T_{\mathrm{B}}$.

$X_{\mathrm{F}}=X_{0}-V_{\mathrm{F}} T$,

where $X_{0}$ is the initial position of the front with respect to the domain at $T=0$ and $V_{\mathrm{F}}$ is the net frontal velocity.

$V_{\mathrm{F}}=U_{\mathrm{tr}}+U_{\mathrm{F}}$.

$U_{\mathrm{F}}$ is the frontal speed in the $x$ direction and $U_{\mathrm{tr}}$ is the translation speed in the $x$ direction. Representative values for $U_{\mathrm{F}}$ and $U_{\mathrm{tr}}$ are $1.25 \mathrm{~m} \mathrm{~s}^{-1}$ and $-0.25 \mathrm{~m} \mathrm{~s}^{-1}$ respectively. For $T_{\mathrm{B}} \geq T \leq T_{\mathrm{S}}$.

$$
\begin{aligned}
X_{\mathrm{F}} & =X_{0}-V_{\mathrm{F}} \\
& \times\left[T_{\mathrm{B}}+\left(T-T_{\mathrm{B}}\right)\left(1-0.5\left(\frac{T-T_{\mathrm{B}}}{T_{\mathrm{S}}-T_{\mathrm{B}}}\right)\right)\right] .
\end{aligned}
$$


For $T_{\mathrm{s}}<T<0$,

$X_{\mathrm{F}}=X_{0}-V_{\mathrm{F}}\left(T_{\mathrm{B}}+0.5\left(T_{\mathrm{S}}-T_{\mathrm{B}}\right)\right)$,

which implies that the front will remain stationary for $20 \mathrm{~h}$. Finally, when $T>0$,

$$
\begin{aligned}
X_{\mathrm{F}} & =X_{0}-V_{\mathrm{F}}\left(T_{\mathrm{B}}+0.5\left(T_{\mathrm{S}}-T_{\mathrm{B}}\right)\right) \\
& +U_{\mathrm{F}} T .
\end{aligned}
$$

\section{REFERENCES}

Bateman, H. 1954. Table of integral transforms, Vol. 1. McGraw-Hill, New York, 391 pp.

Blumen, W. 1972. Geostrophic adjustment. Rev. Geophys. Space Phys. 10, 485-528.

Bolin, B. 1953. The adjustment of a nonbalanced velocity field towards geostrophic equilibrium in a stratified fluid. Tellus 5, 373-385.

Geisler, J. E. 1970. Linear theory of the response of a two-layer ocean to a moving hurricane. Geophys. Fluid Dyn. 1, 249-272.

Hellerman, S. 1967. An updated estimate of the wind stress on the world ocean. Mon.Wea. Rev. 95, 607-626.

Nihoul, J. C. J. (ed.) 1980. Marine turbulence. Elsevier Scientific Publishing Co., Amsterdam, The Netherlands, $378 \mathrm{pp}$.

Ogura, Y. and Phillips, N. A. 1962. Scale analysis of deep and shallow convention in the atmosphere. $J$. Atmos. Sci. 19, 173-179.

Orlanski, I. and Ross, B. B. 1973. Numerical simulation of the generation and breaking of internal gravity waves. J. Geophys. Res. 78, 8808-8826.

Orlanski, I., Ross, B. B. and Polinsky, L. J. 1974. Diurnal variation of the planetary boundary layer in a mesoscale model. J. Atmos. Sci. 31, 965-989.

Orlanski, I. 1975. A rational subdivision of scales for atmospheric processes. Bull. Amer. Meteorol. Soc. 56, $527-530$.
Orlanski, I. 1976. A simple boundary condition for unbounded hyperbolic flows. J. Comput. Phys. 21, 251-269.

Orlanski, I. and Ross, B. B. 1977. The circulation association with a cold front. Part I: Dry case. $J$. Atmos. Sci. 34, 1619-1633.

Pollard, R. T. 1968. On the generation by winds of intertial waves. Ph.D. Thesis, St. John's College of the University of Cambridge, Cambridge, England.

Pollard, R. T. 1970. On the generation by winds of intertial waves in the ocean. Deep Sea Res. 17, 795-812.

Ross, B. B. and Orlanski, I. 1978. The circulation association with a cold front. Part II: Moist case. $J$. Atmos. Sci. 35, 445-465.

Rossby, C.-G. 1937. On the mutual adjustment of pressure and velocity distributions in certain simple current systems, 1.J. Mar. Res. 1, 15-28.

Rossby, C.-G. 1938. On the mutual adjustment of pressure and velocity distributions in certain simple current systems, 2, J. Mar. Res. 1, 239-262.

Tepper, M. 1955. On the generation of pressure jump lines by impulsive addition of momentum to simple current systems. J. Meteorol. 12, 287-297.

Veronis, G. and Stommel, H. 1956. The action of variable wind stresses on a stratified ocean. J. Mar. Res. 15, 43-75. 